University of Nebraska - Lincoln

DigitalCommons@University of Nebraska - Lincoln

$12-2013$

\title{
Imaging coherent electronic motion in atoms by ultrafast electron diffraction
}

Hua-Chieh Shao

University of Nebraska-Lincoln, hshao@unl.edu

Anthony F. Starace

University of Nebraska-Lincoln, astarace1@unl.edu

Follow this and additional works at: https://digitalcommons.unl.edu/physicsstarace

Shao, Hua-Chieh and Starace, Anthony F., "Imaging coherent electronic motion in atoms by ultrafast electron diffraction" (2013). Anthony F. Starace Publications. 204.

https://digitalcommons.unl.edu/physicsstarace/204

This Article is brought to you for free and open access by the Research Papers in Physics and Astronomy at DigitalCommons@University of Nebraska - Lincoln. It has been accepted for inclusion in Anthony F. Starace Publications by an authorized administrator of DigitalCommons@University of Nebraska - Lincoln. 


\title{
Imaging coherent electronic motion in atoms by ultrafast electron diffraction
}

\author{
Hua-Chieh Shao and Anthony F. Starace \\ Department of Physics and Astronomy, The University of Nebraska, Lincoln, Nebraska 68588-0299, USA
}

(Received 14 October 2013; published 26 December 2013)

\begin{abstract}
Ultrafast electron diffraction from time-varying coherent electronic states of the $\mathrm{H}$ atom is analyzed theoretically. This theoretical analysis identifies the conditions necessary to obtain time-resolved measurements. Electron diffraction from coherent electronic states exhibiting breathing and wiggling modes of electronic motion are simulated numerically in order to demonstrate the capability of attosecond electron pulses to image electron dynamics. The scattering patterns and their temporal behaviors are shown to differentiate the two kinds of target electronic motion. Moreover, our simulations show that inelastic processes contribute significantly to the diffraction patterns. Thus, although the diffraction patterns clearly distinguish different modes of target electronic motion, they cannot be easily related to the time-dependent target charge density.
\end{abstract}

DOI: 10.1103/PhysRevA.88.062711

PACS number(s): 03.65.Nk, 34.80.Dp, 34.80.Pa

\section{INTRODUCTION}

The past decade has seen an expansion of interest in ultrafast processes, from a focus on femtosecond molecular dynamics $[1,2]$ to the investigation of attosecond electron dynamics [3-6], owing both to the key role played by electronic motion in reactions and to technological advances that have enabled the production of ultrashort extreme ultraviolet, $x$ ray, and electron pulses. In order to understand reaction mechanisms, direct imaging and visualization of electronic motion provides insight into electron dynamics, such as, for example, the interplay between the electronic and nuclear degrees of freedom in chemical reactions. Imaging such reaction dynamics requires stringent spatial and temporal resolutions. Ultrafast electron and $\mathrm{x}$-ray pulses have for a long time been used to study atomic and molecular structures owing to their (sub-)angstrom resolution. Nowadays, femtosecond x-ray [7] and electron pulses [8] have been achieved, and the feasibility of going beyond the femtosecond domain to the attosecond regime, which is the typical time scale for electronic motion, are forefront topics of research.

Ultrafast electron diffraction and microscopy have been used to investigate the reaction dynamics in molecular and condensed-matter systems using various pump-probe schemes [8-12]. The achievable temporal resolutions have been improved steadily. At present, electron pulses with sub-100-fs duration are available [13]. However, most commonly in such investigations an independent atom approximation is assumed; i.e., the electronic degrees of freedom are frequently neglected. Moreover, in the experiments that have so far been carried out, the pulse durations of the electron pulses typically range from several picoseconds to sub-picoseconds, which may be insufficient to resolve the electronic motion. Hence, changes in electronic structures have to be inferred indirectly, which makes the roles played by electrons in these reactions ambiguous. Recently, however, it has been proposed to produce attosecond electron pulses by compressing electron pulses using a microwave cavity [14,15] or an optical pulse $[16,17]$. Moreover, simulations have demonstrated the ability of attosecond electron pulses to image electron motions in atoms and molecules in both coordinate $[3,18,19]$ and momentum space [20].
Although these experimental and theoretical developments have raised expectations concerning the ability of attosecond electron pulses to image electronic motion in target atoms and molecules, a recent simulation of imaging such motion with ultrashort $\mathrm{x}$-ray pulses has indicated that, contrary to expectation, the motion that is measured is not that of the time-dependent target charge density [21]. Specifically, conventional theory formulates $\mathrm{x}$-ray diffraction as elastic scattering from the target charge density. The scattering intensity $I$ is proportional to the absolute square of the target form factor, which is the Fourier transform of the target charge density,

$$
I(s) \propto\left|\int d x^{\prime} e^{i s \cdot x^{\prime}} \rho\left(x^{\prime}\right)\right|^{2},
$$

where $s$ is the momentum transfer. Therefore, the charge density can be obtained by analyzing x-ray diffraction images, and a number of algorithms for retrieving the target structure are based on this interpretation [22]. However, if the target electronic states are nonstationary, Dixit et al. [21] showed that the diffraction patterns for 1 -fs $\mathrm{x}$-ray pulses have little relation to the target charge densities. In addition, radiation damage to the target electronic structure was found to be significant. Specifically, they showed that because of the finite bandwidth $(0.5 \mathrm{eV})$ of the 1-fs x-ray pulses, inelastic transitions from the time-dependent target coherent state to states whose energy levels are within this bandwidth cannot be resolved in diffraction experiments. As a result, these inelastic transitions significantly alter the diffraction patterns from those expected for elastic x-ray scattering, thus obfuscating the interpretation of the target electronic motion from the $\mathrm{x}$-ray diffraction patterns.

In this paper we develop a scattering theory that describes the collision of an ultrafast (attosecond) electron pulse from a coherent superposition state of a target, as shown schematically in Fig. 1. The theory is based on Robicheaux's [23] general description of scattering involving a coherent matter beam. One purpose of developing such a theory is to identify the factors that affect time-resolved measurements of target electronic structures so that optimal conditions can be prescribed for experiments. Another purpose of such a theory 


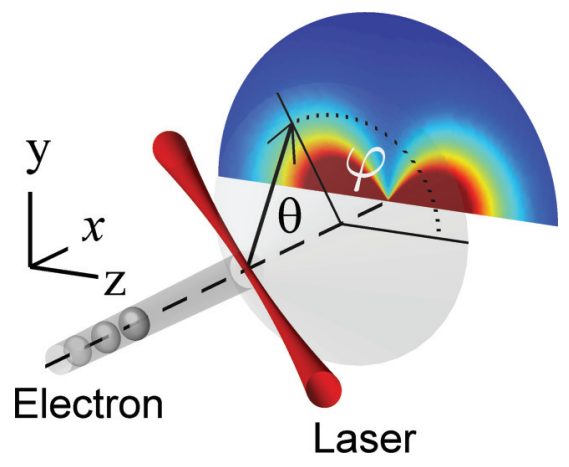

FIG. 1. (Color online) Schematic setup for ultrafast electron diffraction from a coherent superposition state of a target. The coherent state is produced by a pump laser polarized along the $z$ axis. The diffraction patterns are measured as a function of pump-probe delay time. The scattering angle $\theta$ and azimuthal angle $\varphi$ are defined here for future reference.

is to provide a correct interpretation of the results, thereby facilitating analyses aimed at retrieving detailed information about target electronic motion. We illustrate the theory by simulating ultrafast electron diffraction from time-dependent coherent electronic superposition states of the $\mathrm{H}$ atom. Two types of electronic motions are considered: breathing and wiggling. In the first case the coherent state is a superposition of the same-parity $3 p$ and $4 p$ states, as in Ref. [19], whereas in the second case the coherent state is a superposition of the opposite-parity $3 d$ and $4 f$ states, as in Ref. [21]. The breathing mode exhibits localization and delocalization of the electron density, while the wiggling mode manifests the migration of the electron charge from one side of the atom to the other (cf. Fig. 2). These two kinds of electronic motions have important roles in molecular reactions, as in bond making and/or breaking.

This article is structured as follows. Our formulation of the theory for scattering of a coherent electron pulse from a time-dependent coherent target state is presented in Sec. II. Our simulations of the diffraction patterns for two different target coherent states are presented and analyzed in Sec. III. Finally, we summarize our results and present some conclusions in Sec. IV.

\section{THEORY}

The basic idea is to construct coherent wave packets for both the projectile electron pulse and the target in its coherent state and follow their progression in time [23]. Coherence of a system means that one or more of the off-diagonal elements in its density matrix are nonzero. The target coherent state is assumed to be created by some (optical) pump procedure, so that the time delay between its creation and the collision with the incident electron pulse can be well defined. The probabilities for scattering of the incident electron pulse from the target coherent state are calculated using the time-dependent wave packets. The temporal behavior of the target can be investigated by varying the delay time between the (optical) pump and electron probe pulses. The theory identifies the experimental conditions necessary for
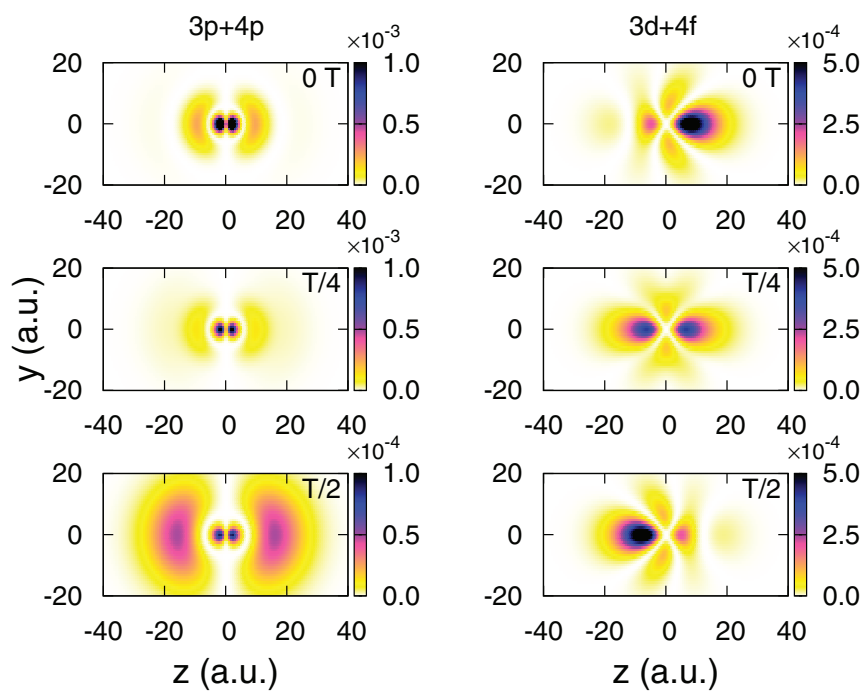

FIG. 2. (Color online) Electron charge densities for two coherent superposition states of the $\mathrm{H}$ atom involving two distinct modes of motion plotted in the $y-z$ plane as a function of time (cf. Fig. 1 for the definition of the coordinates). The left column shows the breathing mode exhibited by an equal superposition of the equal-parity $3 p$ and $4 p$ states; the right column shows the wiggling mode exhibited by an equal superposition of the opposite-parity $3 d$ and $4 f$ states. The period $\mathrm{T}$ of the charge oscillation is $6.25 \mathrm{fs}$ in each case.

obtaining time-resolved scattering signals. A main goal of theory is to specify experimental configurations such that the contributions of the projectile electron wave packet and the target coherent-state wave packet factorize in the expression for the scattering probability so that the diffraction patterns reveal directly characteristic properties of the target state [24]. For scattering from stationary states, factorization can be achieved for sufficiently high collision energy, while for imaging coherent states, more detailed considerations are required. Our presentation of the theory below is separated into analyses of the kinematical and the dynamical aspects.

\section{A. Kinematical aspects}

We consider a projectile electron colliding with a target atom $A$,

$$
e^{-}\left(\boldsymbol{k}_{0}\right)+A\left(\boldsymbol{k}_{1}, n\right) \rightarrow e^{-}\left(\boldsymbol{k}_{a}\right)+A\left(\boldsymbol{k}_{b}, m\right),
$$

where $\boldsymbol{k}_{0}\left(\boldsymbol{k}_{a}\right)$ and $\boldsymbol{k}_{1}\left(\boldsymbol{k}_{b}\right)$ are the momenta of the projectile electron and the target before (after) the collision. The internal states of the target in the entrance and exit channels are specified by the set of quantum numbers $n$ and $m$, respectively. The target has some coherent electronic motion to be probed by the incident electron.

Let $H$ be the Hamiltonian of the colliding system,

$$
H=H_{0}+H_{1}+V,
$$

where $H_{0}$ and $H_{1}$ are the Hamiltonians for the projectile and the target, respectively, and $V$ is the potential describing the interaction between them. The eigenstate $\psi_{i}^{(+)}$of $H$ satisfying outgoing wave boundary conditions may be written as

$$
\psi_{i}^{(+)}=\psi_{i}+\mathscr{G}^{(+)}\left(\varepsilon_{i}\right) V \psi_{i},
$$


where the subscript $i \equiv\left\{\boldsymbol{k}_{0}, \boldsymbol{k}_{1}, n\right\}$ specifies the state of the entrance channel, $\psi_{i}$ is the corresponding eigenstate of $H_{0}+$ $H_{1}, \mathscr{G}^{(+)}(\varepsilon)$ is the Green's function defined by

$$
\mathscr{G}^{(+)}(\varepsilon)=\frac{1}{\varepsilon-H+i \alpha},
$$

and $\varepsilon_{i}$ is the energy eigenvalue for the eigenstate $\psi_{i}^{(+)}$,

$$
H \psi_{i}^{(+)}=\varepsilon_{i} \psi_{i}^{(+)} .
$$

The energy $\varepsilon_{i}$ is the sum of the kinetic energy $E_{0}$ of the incident electron, the kinetic energy $E_{1}$ of the target, and the internal energy $\omega_{n}$ of the target, i.e.,

$$
\varepsilon_{i}=E_{0}+E_{1}+\omega_{n} .
$$

At time $t=0$ the coherent state of the system is represented as a product of projectile and target wave packets,

$$
\psi_{\mathrm{coh}}^{(+)}=\int d \boldsymbol{k}_{0} d \boldsymbol{k}_{1} a_{0}\left(\boldsymbol{k}_{0}\right) a_{1}\left(\boldsymbol{k}_{1}\right) \sum_{n} C_{n} \psi_{i}^{(+)},
$$

where $a_{0}\left(\boldsymbol{k}_{0}\right)$ and $a_{1}\left(\boldsymbol{k}_{1}\right)$ are the respective momentum amplitudes of the projectile and the target, $C_{n}$ is the amplitude of the internal state $n$ of the target, and $\psi_{i}^{(+)}$satisfies Eq. (6). Unlike conventional treatments, in which the wave packet of the target is neglected, here the target is also represented as a wave packet. As discussed below, this is necessary in order to correctly describe the time-dependent scattering process. The time-dependent wave packet $\psi_{\mathrm{coh}}^{(+)}(t)$ can be obtained by applying the time-evolution operator $e^{-i H t}$ to both sides of Eq. (8) and using Eq. (6) to obtain

$$
\psi_{\mathrm{coh}}^{(+)}(t)=\sum_{n} C_{n} \int d \boldsymbol{k}_{0} d \boldsymbol{k}_{1} a_{0} a_{1} e^{-i \varepsilon_{i} t} \psi_{i}^{(+)},
$$

where the interchangeability of $e^{-i H t}$ and the wave-packet integrals is assumed. Throughout this article we use atomic units (a.u.), $\hbar=1, e=1$, and $m_{e}=1$, although $m_{e} \equiv m_{a}$ is included in our equations in order to show the dependence on projectile mass explicitly.

Let $\psi_{f}$ be the eigenstate of the final-state channel with energy $\varepsilon_{f}$, where $f \equiv\left\{\boldsymbol{k}_{a}, \boldsymbol{k}_{b}, m\right\}$ labels the channel. The transition amplitude $\mathscr{A}_{f}^{(+)}(t)$ to this final state equals the projection of $\psi_{\text {coh }}^{(+)}(t)$ onto $\psi_{f}$ :

$$
\mathscr{A}_{f}^{(+)}(t)=\left(\psi_{f}, \psi_{\mathrm{coh}}^{(+)}(t)\right) .
$$

Substituting Eq. (9) into Eq. (10) and using the definitions of $\psi_{i}^{(+)}$in Eq. (4) and of $\mathscr{G}^{(+)}(\varepsilon)$ in Eq. (5), one obtains (see, e.g., pp. 78-79 of Ref. [25] or pp. 525-526 of Ref. [26]),

$$
\begin{aligned}
\mathscr{A}_{f}^{(+)}(t)= & \sum_{n} C_{n} \int d \boldsymbol{k}_{0} d \boldsymbol{k}_{1} a_{0}\left(\boldsymbol{k}_{0}\right) a_{1}\left(\boldsymbol{k}_{1}\right) \\
& \times \frac{e^{-i \varepsilon_{i} t}}{\varepsilon_{i}-\varepsilon_{f}+i \alpha}\left(\psi_{f}, \mathscr{T} \psi_{i}\right),
\end{aligned}
$$

where

$$
\mathscr{T}=V+V \mathscr{G}^{(+)}\left(\varepsilon_{i}\right) V .
$$

Since the total linear momentum is conserved in the collision process if no external field is present, a $\delta$ function representing the conservation of linear momentum can be factored out from the transition matrix. Accordingly, set

$$
\left(\psi_{f}, \mathscr{T} \psi_{i}\right)=\delta\left(\mathcal{P}_{f}-\mathcal{P}_{i}\right) T_{f i},
$$

where $\mathcal{P}_{i} \equiv \boldsymbol{k}_{0}+\boldsymbol{k}_{1}$ and $\mathcal{P}_{f} \equiv \boldsymbol{k}_{a}+\boldsymbol{k}_{b}$ are the linear momenta of the reactants and products. In most experiments, measurements are performed in the asymptotic region, and the asymptotic transition probability density $\left|\mathscr{A}_{f}\right|^{2}$ is defined by the following limit:

$$
\left|\mathscr{A}_{f}\right|^{2} \equiv \lim _{\alpha \rightarrow 0^{+}} \lim _{t \rightarrow+\infty}\left|\mathscr{A}_{f}^{(+)}(t)\right|^{2} .
$$

Substituting Eqs. (11) and (13) into Eq. (14) and taking the limits, which give $\delta$ functions in energy (see, e.g., pp. 630-633 of Ref. [26]), one obtains

$$
\begin{aligned}
\left|\mathscr{A}_{f}\right|^{2}= & \sum_{n^{\prime} n} C_{n^{\prime}}^{*} C_{n} \int d \boldsymbol{k}_{0}^{\prime} d \boldsymbol{k}_{1}^{\prime} d \boldsymbol{k}_{0} d \boldsymbol{k}_{1} a_{0}^{*}\left(\boldsymbol{k}_{0}^{\prime}\right) a_{0}\left(\boldsymbol{k}_{0}\right) \\
& \times a_{1}^{*}\left(\boldsymbol{k}_{1}^{\prime}\right) a_{1}\left(\boldsymbol{k}_{1}\right)(2 \pi)^{2} \delta\left(\varepsilon_{f}-\varepsilon_{i^{\prime}}\right) \delta\left(\varepsilon_{f}-\varepsilon_{i}\right) \\
& \times \delta\left(\mathcal{P}_{f}-\mathcal{P}_{i^{\prime}}\right) \delta\left(\mathcal{P}_{f}-\mathcal{P}_{i}\right) T_{f i^{\prime}}^{*} T_{f i},
\end{aligned}
$$

where $i^{\prime} \equiv\left\{\boldsymbol{k}_{0}^{\prime}, \boldsymbol{k}_{1}^{\prime}, n^{\prime}\right\}$. Note that $a_{0}^{*}\left(\boldsymbol{k}_{0}^{\prime}\right) a_{0}\left(\boldsymbol{k}_{0}\right)$ is the density matrix element $\rho_{0}\left(\boldsymbol{k}_{0}^{\prime}, \boldsymbol{k}_{0}\right)$ of the projectile; likewise, $\rho_{1}\left(\boldsymbol{k}_{1}^{\prime}, n^{\prime} ; \boldsymbol{k}_{1}, n\right)=C_{n^{\prime}}^{*} C_{n} \otimes a_{1}^{*}\left(\boldsymbol{k}_{1}^{\prime}\right) a_{1}\left(\boldsymbol{k}_{1}\right)$ is the direct product of the internal and external parts of the density matrix element for the target. As shown in Eq. (15), each of the components of the coherent wave packet that contributes to the probability density $\left|\mathscr{A}_{f}\right|^{2}$ obeys both energy and momentum conservation. As noted when we constructed the wave packet $\psi_{\text {coh }}^{(+)}$in Eq. (8), if the target were represented by a plane wave instead of by a wave packet, one would find that conservation of momentum and energy enforces the equalities $\boldsymbol{k}_{0}=\boldsymbol{k}_{0}^{\prime}$ and $n=n^{\prime}$ in Eq. (15). Therefore, no off-diagonal terms of the density matrices $\rho_{0}$ and $\rho_{1}$ would contribute to $\left|\mathscr{A}_{f}\right|^{2}$. As discussed below, such a result would prevent one from resolving the time-dependent motion of the coherent superposition state of the target.

The transition probability $\mathscr{P}$ in an experiment is the sum of all final states that are detected,

$$
\mathscr{P}=\sum_{m} \int d \boldsymbol{k}_{a} d \boldsymbol{k}_{b}\left|\mathscr{A}_{f}\right|^{2},
$$

where the ranges of the integrations over the final-state momenta and of the summation over the target final-state $m$ are determined by the specifics of the experiment. Here we consider that the momentum $\boldsymbol{k}_{b}$ of the target and the kinetic energy $E_{a}$ of the scattered electron are not resolved in experiments. Accordingly, integrations over these variables must be performed.

Owing to the $\delta$ functions in Eq. (15), some integrals can be done straightforwardly when evaluating the transition probability $\mathscr{P}$. First, we use the identity

$$
\delta(x-y) \delta(x-z)=\delta(y-z) \delta(x-z)
$$


to rewrite the products of energy and momentum $\delta$ functions. Then, the $\boldsymbol{k}_{b}, \boldsymbol{k}_{1}^{\prime}$, and $E_{a}$ integrals are performed using these momentum and energy $\delta$ functions. Finally, the remaining $\delta$ function, $\delta\left(\varepsilon_{i^{\prime}}-\varepsilon_{i}\right)$, can be rewritten in terms of the initial momenta using the relation

$$
\delta(f(x))=\left|\frac{d f\left(x_{0}\right)}{d x}\right|^{-1} \delta\left(x-x_{0}\right),
$$

where $x_{0}$ is the root of the function $f$. The expression for the root of the equation $\varepsilon_{i^{\prime}}=\varepsilon_{i}$ can be simplified if one notes that in ultrafast electron diffraction the incident electron is usually very energetic, is well collimated, and has a small spread in energy. Also, the target mass $m_{b}$ is much larger than the electron mass $m_{a}$. Therefore, the kinetic energy $E_{1}$ of the target and the transverse momentum components $\boldsymbol{k}_{0 \perp}$ and $\boldsymbol{k}_{0 \perp}^{\prime}$ of the incident electron (with respect to the central momentum direction of the incident electron wave packet) can be neglected in $\varepsilon_{i}$ and $\varepsilon_{i^{\prime}}$. Evaluating the root using these assumptions, one obtains

$$
\delta\left(\varepsilon_{i^{\prime}}-\varepsilon_{i}\right) \simeq \frac{1}{\left|\boldsymbol{v}_{0}-\boldsymbol{v}_{1}\right|} \delta\left(k_{0 \|}^{\prime}-k_{0 \|}+\Delta k\right),
$$

where the subscripts $\|$ denote the longitudinal components of $\boldsymbol{k}_{0}$ and $\boldsymbol{k}_{0}^{\prime}, \boldsymbol{v}_{0}$ and $\boldsymbol{v}_{1}$ are the respective central velocities of the projectile electron and target wave packets, and

$$
\Delta k \equiv \frac{\omega_{n^{\prime}}-\omega_{n}}{\left|\boldsymbol{v}_{0}\right|}
$$

If $n \neq n^{\prime}$, then $k_{0 \|}$ is shifted by $\Delta k$ with respect to $k_{0 \|}^{\prime}$ in the measured transition probability $\mathscr{P}$ owing to conservation of energy.

Introducing the differential probability $d \mathscr{P} / d \hat{\boldsymbol{k}}_{a}$ to represent the scattering intensity [21] and integrating over $k_{0 \|}^{\prime}$, Eq. (16) becomes

$$
\begin{aligned}
\frac{d \mathscr{P}}{d \hat{\boldsymbol{k}}_{a}} \simeq & \sum_{m} \sum_{n^{\prime} n} C_{n^{\prime}}^{*} C_{n} \int d \boldsymbol{k}_{0 \perp}^{\prime} d \boldsymbol{k}_{0} d \boldsymbol{k}_{1} a_{0}^{*}\left(k_{0 \|}-\Delta k, \boldsymbol{k}_{0 \perp}^{\prime}\right) \\
& \times a_{0}\left(\boldsymbol{k}_{0}\right) a_{1}^{*}\left(k_{1 \|}+\Delta k,\left(\boldsymbol{k}_{0}+\boldsymbol{k}_{1}-\boldsymbol{k}_{0}^{\prime}\right)_{\perp}\right) a_{1}\left(\boldsymbol{k}_{1}\right) \\
& \times(2 \pi)^{2} \frac{m_{a}\left|\boldsymbol{k}_{a}\right|}{\left|\boldsymbol{v}_{0}-\boldsymbol{v}_{1}\right|} T_{f i^{\prime}}^{*} T_{f i} .
\end{aligned}
$$

Equation (21) shows how the off-diagonal elements of $\rho_{0}$ and $\rho_{1}$ interfere with each other when the target is in a nonstationary state. If the target is in a stationary state, the above expression reduces to the usual expression in electron scattering theory (see, e.g., [25]).

To further simplify Eq. (21), we note that for ultrafast electron diffraction, $T_{f i}$ and $\left|\boldsymbol{k}_{a}\right|$ are usually insensitive to the spread of momenta in the incident electron wave packet. Hence, they can be factored out from the integrals. The transition matrix $T_{f i}$ is then approximated by evaluating it at the central momenta $\boldsymbol{p}_{0}$ and $\boldsymbol{p}_{1}$ of the projectile electron and target wave packets. Specifically, we assume

$$
T_{f i} \simeq T_{f \bar{i}} \equiv T_{m n} \quad \text { and } \quad T_{f i^{\prime}} \simeq T_{f \bar{i}^{\prime}} \equiv T_{m n^{\prime}},
$$

where $\bar{i} \equiv\left\{\boldsymbol{p}_{0}, \boldsymbol{p}_{1}, n\right\}, \bar{i}^{\prime} \equiv\left\{\boldsymbol{p}_{0}, \boldsymbol{p}_{1}, n^{\prime}\right\}$, and $f \equiv\left\{\boldsymbol{k}_{a}, \boldsymbol{k}_{b}, m\right\}$. Note that since $\boldsymbol{k}_{a}$ and $\boldsymbol{k}_{b}$ are fixed by energy and momentum conservation once we employ the central momenta $\boldsymbol{p}_{0}$ and $\boldsymbol{p}_{1}$ to evaluate the transition matrix $T_{f i}$, we have replaced the finalstate index " $f$ " in Eq. (22) simply by the final internal state $m$ of the target. Also, the momentum width of the amplitude $a_{1}\left(\boldsymbol{k}_{1}\right)$ of the (well-localized) target is assumed to be much larger than that of the incident electron [25], i.e., the variations of $k_{1 \|}$ and $\boldsymbol{k}_{1 \perp}$ are much larger than those of $k_{0 \|}$ (and hence of $\Delta k)$ and $\boldsymbol{k}_{0 \perp}$; therefore, within the wave-packet integrals we can make the approximation

$$
a_{1}\left(k_{1 \|}+\Delta k,\left(\boldsymbol{k}_{0}+\boldsymbol{k}_{1}-\boldsymbol{k}_{0}^{\prime}\right)_{\perp}\right) \simeq a_{1}\left(\boldsymbol{k}_{1}\right) .
$$

Accordingly, the integral over $\boldsymbol{k}_{1}$ can now be done. We emphasize that this approximation essentially assumes the localization of the target in space. The differential probability can thus be rewritten compactly as

$$
\frac{d \mathscr{P}}{d \hat{\boldsymbol{k}}_{a}} \simeq(2 \pi)^{2} \sum_{m} \sum_{n^{\prime} n} \mathcal{B}_{n^{\prime} n} C_{n^{\prime}}^{*} C_{n} \frac{m_{a}\left|\boldsymbol{k}_{a}\right|}{\left|\boldsymbol{v}_{0}-\boldsymbol{v}_{1}\right|} T_{m n^{\prime}}^{*} T_{m n},
$$

where we have defined the coefficients $\mathcal{B}_{n^{\prime} n}$ as

$$
\mathcal{B}_{n^{\prime} n} \equiv \int d \boldsymbol{k}_{0 \perp}^{\prime} d \boldsymbol{k}_{0} a_{0}^{*}\left(k_{0 \|}-\Delta k, \boldsymbol{k}_{0 \perp}^{\prime}\right) a_{0}\left(\boldsymbol{k}_{0}\right),
$$

where the dependence on $n^{\prime}$ and $n$ originates from $\Delta k$ [see Eq. (20)]. Note that information about the internal structure of the target is embedded in the amplitudes $C_{n}$ and the transition matrix elements $T_{m n}$. The coefficients $\mathcal{B}_{n^{\prime} n}$ describe the coupling between different momentum components of the projectile electron and the target internal state through $\Delta k$, defined in Eq. (20). This coupling can complicate the retrieval of information about the target structure and, hence, of the target electronic motion. This complication dissipates, however, if $\mathcal{B}_{n^{\prime} n}$ does not depend sensitively on the particular states $n$ and $n^{\prime}$. In that case, the properties of the projectile electron may be factored out, so that the differential probability becomes directly proportional to the properties of the initial target state; i.e.,

$$
\frac{d \mathscr{P}}{d \hat{\boldsymbol{k}}_{a}} \propto \sum_{m}\left|\sum_{n} C_{n} T_{m n}\right|^{2}
$$

From Eq. (25), we see that this desired condition holds if the longitudinal momentum width $\Delta p_{0 \|}$ of the incident electron is much larger than $\Delta k$ so that we may set

$$
a_{0}\left(k_{0 \|}-\Delta k, \boldsymbol{k}_{0 \perp}^{\prime}\right) \simeq a_{0}\left(k_{0 \|}, \boldsymbol{k}_{0 \perp}^{\prime}\right)
$$

within the wave-packet integrals.

The physical meaning of Eq. (27) may be stated simply once we define two characteristic times. The momentum and spatial widths of the incident electron pulse must satisfy the usual uncertainty relation, $\Delta p_{0 \|} \Delta x_{0 \|} \geqslant 1 / 2$. Thus, the pulse duration as it crosses the position of the target is given by

$$
\tau_{0}=\frac{\Delta x_{0 \|}}{\left|\boldsymbol{v}_{0}\right|} \geqslant \frac{1}{2 \Delta p_{0 \|}\left|\boldsymbol{v}_{0}\right|}
$$

On the other hand, the period of the electronic motion of the target initial state is given by

$$
\tau_{1}=\frac{2 \pi}{\left|\omega_{n^{\prime}}-\omega_{n}\right|}=\frac{2 \pi}{|\Delta k|\left|v_{0}\right|}
$$


where the second equality follows from Eq. (20). In order for our incident electron pulse to resolve the time-dependent motion of the target initial state, we must therefore require that $\tau_{0} \ll \tau_{1}$. Applying this inequality to the right-hand sides of Eqs. (28) and (29), we obtain that $\Delta p_{0 \|} \gg|\Delta k|$, which is the physical basis for the relation in Eq. (27).

In order to further elucidate the physical meaning of these inequalities, consider the opposite inequality, i.e., that $\Delta p_{0 \|} \ll|\Delta k|$ (i.e., $\tau_{0} \gg \tau_{1}$ ). From Eq. (25), we see that in this case the two wave-packet amplitudes for the incident electron pulse are unlikely to overlap, since the shift in one of them due to $\Delta k$ is much larger than the spread of momenta in $k_{0 \|}$. Hence, $\mathcal{B}_{n^{\prime} n} \approx 0$ for $n^{\prime} \neq n$. Effectively, $\mathcal{B}_{n^{\prime} n}$ resembles a Kronecker $\delta$ function in this case. Accordingly, the differential probability is proportional to the sum of the differential probabilities of the constituent eigenstates weighted by their population, i.e.,

$$
\frac{d \mathscr{P}}{d \hat{\boldsymbol{k}}_{a}} \propto \sum_{m n}\left|C_{n}\right|^{2}\left|T_{m n}\right|^{2} .
$$

Since the differential probability in this case does not involve the relative phases between different transition matrix elements (or scattering amplitudes), only the population of each constituent state (i.e., the diagonal elements of $\rho_{1}$ ) can be measured in experiments. In other words, the target time dependence (or its electronic motion) cannot be observed.

To summarize the above analysis, we have shown that the coherence, both transverse and longitudinal, of the incident electron pulse determines the information that can be retrieved from ultrafast electron diffraction experiments (see also the discussion in Ref. [27]). The transverse coherence determines the image quality $[13,22]$. Namely, the diffraction patterns originate from interference of the scattering amplitudes, so the coherence of the incident pulse across the plane impinging upon the target influences the sharpness of the diffraction pattern and hence the quality of the target structure determination. The longitudinal coherence determines the temporal resolution of the target electronic motion. As discussed above, the momentum width in the longitudinal direction limits the pulse duration. Also, as shown by the $\delta$ function in Eq. (19), the target structure [introduced by $\Delta k$; cf. Eq. (20)] leads to interference of different longitudinal momentum components of the incident electron pulse, which affects the differential probability given in Eq. (21) that describes the time-resolved diffraction patterns. Therefore, the relative phase between the momentum components can, in general, affect the temporal resolution.

The time-dependent phases of the amplitudes $C_{n}$ describe the temporal evolution of the coherent superposition state of the target. In pump-probe schemes, the amplitudes $C_{n}$ depend on how the coherent state is produced. Due to the velocity mismatch between the optical pump and electronic probe pulses, in general, $C_{n}$ is a function of the target position in a gas ensemble. This spatial inhomogeneity affects the temporal resolution. We note, however, that techniques have been developed to reduce such effects $[8,28]$. Therefore, in our simulations the effects of velocity mismatch are neglected, and hence the functional form of $C_{n}$ is taken as

$$
C_{n}=c_{n} e^{-i \omega_{n} t_{d}},
$$

where $t_{d}$ is the pump-probe delay time, and $c_{n}$ is the amplitude at the moment the coherent state is created. The phase of $C_{n}$, which is associated with the temporal behavior of the coherent state, can thus be measured by varying the pump-probe delay time.

\section{B. Dynamical aspect}

In Sec. II A we obtained in Eq. (21) an expression for the differential probability in terms of the transition matrix elements $T_{f i}$ based primarily on the kinematics of the collision process. In this section we focus on the dynamical problem: the evaluation of the matrix elements $T_{f i}$. It is preferable for the incident electron pulse to have a high kinetic energy $(\approx$ tens of $\mathrm{keV}$ ) in order that its de Broglie wavelength is small compared to the target structure and in order to simplify the analysis of the scattering process. For such high electron energies the first-order Born approximation is adequate to describe the scattering from most targets [29]. Also, the effects of exchange of the incident and the target electrons can be neglected [30]. In the first-order Born approximation, the transition matrix element in Eqs. (11) and (12) is given by

$$
\mathscr{T}_{f i} \simeq\left(\chi_{a} \chi_{b} \phi_{m}, V \chi_{0} \chi_{1} \phi_{n}\right),
$$

where $\chi_{i}, i \in\{0, a\}$ (or $i \in\{1, b\}$ ), is the plane wave for the electron (or for the target), and $\phi_{n}$ is the eigenstate of the target. The potential between the projectile electron and the atomic target with atomic number $z$ is

$$
V=-\frac{Z}{\left|\boldsymbol{x}_{0}-\boldsymbol{x}_{\mathrm{nuc}}\right|}+\sum_{i=1}^{Z} \frac{1}{\left|\boldsymbol{x}_{0}-\boldsymbol{r}_{i}\right|},
$$

where $\boldsymbol{x}_{0}, \boldsymbol{x}_{\mathrm{nuc}}$, and $\boldsymbol{r}_{i}$ are the coordinates, respectively, of the projectile electron, the nucleus, and the target electrons. In particular, when the plane waves are written explicitly in Eq. (32), one has

$$
\begin{aligned}
\mathscr{T}_{f i} \simeq & \frac{1}{(2 \pi)^{6}} \int d \boldsymbol{x}_{0} d \boldsymbol{x}_{1} d\left\{\boldsymbol{r}_{i}\right\} e^{-i\left(\boldsymbol{k}_{a} \cdot \boldsymbol{x}_{0}+\boldsymbol{k}_{b} \cdot \boldsymbol{x}_{1}\right)} \\
& \times e^{i\left(\boldsymbol{k}_{0} \cdot \boldsymbol{x}_{0}+\boldsymbol{k}_{1} \cdot \boldsymbol{x}_{1}\right)} \phi_{m}^{*}\left(\left\{\boldsymbol{r}_{i}\right\}\right) V \phi_{n}\left(\left\{\boldsymbol{r}_{i}\right\}\right),
\end{aligned}
$$

where $\boldsymbol{x}_{1}$ is the center-of-mass position of the target and $\left\{\boldsymbol{r}_{i}\right\}$ denotes the collection of target electron coordinates. Since the total linear momentum is conserved, $\delta\left(\mathcal{P}_{f}-\mathcal{P}_{i}\right)$ can be factored out from $\mathscr{T}_{f i}$ [cf. Eq. (13)] to obtain

$$
T_{m n} \simeq \frac{1}{(2 \pi)^{3}} \int d \boldsymbol{y} d\left\{\boldsymbol{y}_{i}\right\} e^{i s \cdot \boldsymbol{y}} \phi_{m}^{*} V \phi_{n},
$$

where $\boldsymbol{s} \equiv \boldsymbol{k}_{0}-\boldsymbol{k}_{a}$ is the momentum transfer, $\boldsymbol{y} \equiv \boldsymbol{x}_{0}-\boldsymbol{x}_{1}$, $\boldsymbol{y}_{i} \equiv \boldsymbol{r}_{i}-\boldsymbol{x}_{1}$, and where the subscripts $f i$ have been simplified to $m n$, as discussed above in Sec. II A. Substituting Eq. (33) into Eq. (35), performing the $y$ integral [29], and using the fact that $m_{a} \ll m_{b}$, one obtains the well-known result (see, e.g., Ref. [30])

$$
T_{m n} \simeq \frac{1}{(2 \pi)^{2}} \frac{2}{\boldsymbol{s}^{2}}\left(-Z \delta_{m n}+\sum_{i=1}^{Z} \int d\left\{\boldsymbol{y}_{i}\right\} e^{i s \cdot y_{i}} \phi_{m}^{*} \phi_{n}\right),
$$


where $\delta_{m n}$ is the Kronecker $\delta$ function. The first term is the scattering from the nucleus (Rutherford scattering); the second term describes the target electronic state transition in the collision.

\section{Formula for the differential probability used in our simulations}

Before presenting our numerical simulations for diffraction of a 10-keV attosecond pulse from coherent states of the $\mathrm{H}$ atom, we must present the actual formula for the differential probability that is used in our simulations. In Sec. II A we derived a fairly accurate expression for the differential probability, given in Eq. (21). We then sketched the necessary conditions or approximations necessary for us to reduce that accurate expression to the one in Eq. (26). This latter result for the differential probability is the most desirable one since it is proportional to the absolute square of the transition matrix element from the coherent initial state of the target, thus making interpretation of the diffraction pattern straightforward. However, the goal of our simulations is to use as accurate an expression for the differential probability as possible to see how close the calculated results are to the desired but more approximate result. For this reason the approximations in Eqs. (22) and (27) are not used in our simulations. In general, this means that we do not assume that the properties of the incident electron pulse and the target can be factorized in the differential probabilities. More specifically, not using Eq. (22) means that we do not factor the transition matrix elements out from under the momentum integrals in Eq. (21); not using Eq. (27) means that the width of the momentum profile of the incident electron pulse is not assumed to be much larger than $\Delta k$ [see Eq. (20) and the sentence below that equation]. However, we do assume that Eq. (23) is valid, i.e., that the momentum width of the target atom is much larger than that of the incident electron pulse. This assumption implies that $T_{f i}$ and $\left|\boldsymbol{k}_{a}\right|$ are insensitive to variations of the momentum $\boldsymbol{k}_{1}$ of the target within the momentum envelope of the incident electron pulse, so that the transition amplitude $T_{f i}$ and the magnitude of the scattered electron momentum $\left|\boldsymbol{k}_{a}\right|$ can be evaluated at the central momentum $\boldsymbol{p}_{1}$ of the target and hence can be removed from the integral in Eq. (21) over the target momentum $\boldsymbol{k}_{1}$. With this assumption, the integral over the target momentum $\boldsymbol{k}_{1}$ can be done analytically. Also, the transition matrix elements $T_{f i}$ and $T_{f i^{\prime}}^{*}$ are evaluated at $\boldsymbol{p}_{1}$ and are functions of $\boldsymbol{k}_{0}$ and $\boldsymbol{k}_{0}^{\prime}$; in other words, $i=\left\{\boldsymbol{k}_{0}, \boldsymbol{p}_{1}, n\right\}$ and $i^{\prime}=\left\{\boldsymbol{k}_{0}^{\prime}, \boldsymbol{p}_{1}, n^{\prime}\right\}$. Hence, applying these assumptions to Eq. (21), we obtain the following result for the differential probability:

$$
\begin{aligned}
\frac{d \mathscr{P}}{d \hat{\boldsymbol{k}}_{a}} \simeq & \sum_{m} \sum_{n^{\prime} n} C_{n^{\prime}}^{*} C_{n} \int d \boldsymbol{k}_{0 \perp}^{\prime} d \boldsymbol{k}_{0} a_{0}^{*}\left(\boldsymbol{k}_{0}^{\prime}\right) a_{0}\left(\boldsymbol{k}_{0}\right) \\
& \times(2 \pi)^{2} \frac{m_{a}\left|\boldsymbol{k}_{a}\right|}{\left|k_{0 \|} / m_{a}-p_{1 \|} / m_{b}\right|} T_{f i^{\prime}}^{*} T_{f i} .
\end{aligned}
$$

Unless indicated otherwise, this formula for the differential probability is the one used to obtain all numerical simulation results that we present in this paper.

\section{IMAGING TIME-DEPENDENT (COHERENT) STATES OF THE H ATOM}

In order to illustrate the theory presented in Sec. II for diffraction of an ultrafast electron pulse from a time-dependent target electronic state, we present in this section simulations of ultrafast electron pulse scattering from two different coherent superposition states of the $\mathrm{H}$ atom. An additional aim of these simulations is to demonstrate the conditions under which the time-dependent electronic motion of these superposition states can be imaged and also to discuss the proper interpretation of such images. In Sec. III A we present two calculational details applicable to our simulations for an $\mathrm{H}$ atom target: In Sec. III A 1 we present analytic formulas for the transition matrix elements in Eq. (36), and in Sec. III A 2 we describe our approach for approximating the summation over all target inelastic transitions. In Sec. III B we discuss the parameters of the incident electron pulse and the target in our simulations. Finally, in Sec. III C we present results of our simulations for scattering of a 100-as, 10-keV electron pulse from two coherent states of the $\mathrm{H}$ atom target: In Sec. III C 1 we present results for the coherent $3 p+4 p$ state and in Sec. III C 2 we present results for the coherent $3 d+4 f$ state.

\section{A. Calculational details for an $\mathbf{H}$ atom target}

\section{Analytic formulas for the transition matrix elements}

In order to evaluate the integral on the right-hand side of Eq. (36), we express the target wave functions as products of hydrogenic radial functions and spherical harmonics and expand the plane wave $e^{i s \cdot y_{1}}$ in spherical waves so that the radial and angular integrals can be carried out independently to obtain

$$
\int d \boldsymbol{y}_{1} e^{i s \cdot y_{1}} \phi_{n_{f} l_{f} m_{f}}^{*} \phi_{n_{i} l_{i} m_{i}}=\sum_{l m} \mathcal{R}_{l}(|\boldsymbol{s}|) \Theta_{l m}(\hat{\boldsymbol{s}}),
$$

where the subscripts $n, l$, and $m$ of $\phi$ denote, respectively, the principal, orbital, and magnetic quantum numbers of the initial and final target states. The result for the angular integration over $\hat{\boldsymbol{y}}_{1}, \Theta_{l m}(\hat{\boldsymbol{s}})$, was obtained using the Gaunt coefficients, which can be represented in terms of Wigner $3 j$ symbols, and the remaining angular dependence on $\hat{s}$ is given by a spherical harmonic. The result is

$$
\begin{aligned}
\Theta_{l m}(\hat{\boldsymbol{s}})= & i^{l}(-1)^{m_{f}} \sqrt{\frac{(2 l+1)\left(2 l_{i}+1\right)\left(2 l_{f}+1\right)}{4 \pi}} \\
& \times\left(\begin{array}{ccc}
l_{f} & l & l_{i} \\
-m_{f} & m & m_{i}
\end{array}\right)\left(\begin{array}{ccc}
l_{f} & l & l_{i} \\
0 & 0 & 0
\end{array}\right) Y_{l m}^{*}(\hat{\boldsymbol{s}}) .
\end{aligned}
$$

Note that selection rules for the $3 j$ symbols in Eq. (39) greatly restrict the number of nonzero terms in the summation in Eq. (38). The result of the radial integration on the left-hand side of Eq. (38), $\mathcal{R}_{l}(|s|)$, can be written in terms of sums of 
hypergeometric functions ${ }_{2} F_{1}[31]$ :

$$
\begin{aligned}
\mathcal{R}_{l}(|\boldsymbol{s}|)= & \frac{16 \pi}{(2 l+1) ! !} \frac{1}{n_{i}^{2}} \sqrt{\left(n_{i}+l_{i}\right) !\left(n_{i}-l_{i}-1\right) !} \frac{1}{n_{f}^{2}} \sqrt{\left(n_{f}+l_{f}\right) !\left(n_{f}-l_{f}-1\right) !} \sum_{k_{i}=0}^{n_{i}-l_{i}-1} \frac{(-1)^{k_{i}}\left(\frac{2}{n_{i}}\right)^{k_{i}+l_{i}}}{\left(n_{i}-l_{i}-1-k_{i}\right) !\left(2 l_{i}+1+k_{i}\right) ! k_{i} !} \\
& \times \sum_{k_{f}=0}^{n_{f}-l_{f}-1} \frac{(-1)^{k_{f}}\left(\frac{2}{n_{f}}\right)^{k_{f}+l_{f}}}{\left(n_{f}-l_{f}-1-k_{f}\right) !\left(2 l_{f}+1+k_{f}\right) ! k_{f} !} \mu^{l_{i}+l_{f}+k_{i}+k_{f}+3}\left(l+l_{i}+l_{f}+k_{i}+k_{f}+2\right) ! \\
& \times \frac{(\mu|s|)^{l}}{\left[1+(\mu|s|)^{2}\right]^{l_{i}+l_{f}+k_{i}+k_{f}+2}}{ }_{2} F_{1}\left(\frac{l-l_{i}-l_{f}-k_{i}-k_{f}-1}{2}, \frac{l-l_{i}-l_{f}-k_{i}-k_{f}}{2} ; l+\frac{3}{2} ;-(\mu|s|)^{2}\right),
\end{aligned}
$$

where $\mu=n_{i} n_{f} /\left(n_{i}+n_{f}\right)$ and where the magnitude of the momentum transfer, $|\boldsymbol{s}|$, is related to the scattering angle, $\theta$, as follows:

$$
|\boldsymbol{s}|=\sqrt{\boldsymbol{k}_{0}^{2}+\boldsymbol{k}_{a}^{2}-2\left|\boldsymbol{k}_{0}\right|\left|\boldsymbol{k}_{a}\right| \cos \theta} .
$$

\section{Calculational procedure for summing over target final states}

The energy bandwidth of the electron pulse is estimated as $\Delta E_{0} \simeq \Delta p_{0 \|}\left|p_{0 \|}\right| / m_{a} \approx 18.2 \mathrm{eV}$. Owing to this large bandwidth, inelastic transitions to other target states having transition energies within the bandwidth $\Delta E_{0}$ cannot be resolved. Accordingly, these inelastic channels must be included when calculating the differential probabilities. Impact ionization is not considered in our simulations because ionization channels can be easily distinguished from other channels by the different final-state products. Since no closed form expression is available for the transition probability in which the inelastic transitions to all target bound states are summed, the infinite sum over the bound final states $m$ in Eq. (37) must be done approximately. The analytic expressions in Eqs. (39) and (40) are used to evaluate the differential probabilities from the initial coherent superposition state (i.e., $3 p+4 p$ or $3 d+4 f$ ) of the target to all final target states having principal quantum numbers $n_{f} \leqslant 9$. For those high Rydberg states having principal quantum numbers $n_{f}>9$, an extrapolation technique was employed to obtain the differential probabilities to the infinity of those states extending up to the ionization threshold. These extrapolations for states with $n_{f}>9$ were carried out only for states having orbital angular momentum $l_{f} \leqslant 6$. Note that this extrapolation technique is similar to the interpolation technique of McCarroll [32] for obtaining the total excitation cross sections of the $\mathrm{H}$ atom from the ground state to bound states with $n_{f} \geqslant 5$ : The values at each $n_{f} \geqslant 5$ are interpolated between the calculated excitation cross sections to states with $n_{f}=2,3$, and 4 and those for excitation (ionization) to the continuum. However, the method of Ref. [32] cannot be implemented directly in our simulations for two reasons: First, in this paper we are interested in partial differential probabilities and not total probabilities; and second, we have not calculated excitations (ionization) to the continuum. For these reasons we employ an appropriate extrapolation technique.

Note that for our ultrafast electron diffraction problem, the differential scattering probabilities are highly sensitive to the angular momenta of the target initial and final states, as may be seen from Eq. (39). Also, in general, the differential probability is not a smooth function of $l_{f}$. Therefore, any extrapolation method can only be applied to transitions to states with the same final orbital angular momentum. The radial parts of hydrogenic wave functions are known to be smooth functions of the principal quantum number $n_{f}$ for high-level Rydberg states [33]. Thus, the differential probability is also a smooth function of $n_{f}$, ensuring that the errors introduced by an extrapolation procedure are small. More specifically, for each momentum transfer $s$ and for each $l_{f}$, the radial parts $\mathcal{R}_{l}(|s|) / s^{2}$ of the transition matrices [cf. Eqs. (36) and (38)] for each component of the initial target coherent superposition state to Rydberg states with principal quantum numbers $n_{f}=10-13$ are calculated explicitly. Then the transition probabilities to Rydberg states with $n_{f}>13$ (for fixed values of $l_{f}$ and $m_{f}$ ) are extrapolated using a cubic polynomial whose coefficients are determined by the calculated transition matrices for $n_{f}=10-13$. The infinite sum over $n_{f}$ is then approximated by an integration over energy, using the relation $d n_{f} / d \omega_{n_{f}}=n_{f}^{3}$ [32]. The summation over the magnetic quantum number $m_{f}$ (for a given $l_{f}$ ) can be done without difficulty. As noted above, this extrapolation is carried out for all $l_{f} \leqslant 6$. In short, the differential probability to Rydberg states with $n_{f} \geqslant 10$ is approximated as

$$
\begin{aligned}
\frac{d \mathscr{P}_{\mathrm{Ryd}}}{d \hat{\boldsymbol{k}}_{a}} \simeq & (2 \pi)^{2} \sum_{l_{f}} \sum_{m_{f}=-l_{f}}^{l_{f}} \int_{\omega_{10}}^{\omega_{\infty}} d \omega_{n_{f}} \frac{d n_{f}}{d \omega_{n_{f}}} \frac{m_{a}\left|\boldsymbol{k}_{a}\right|}{\left|\boldsymbol{v}_{0}-\boldsymbol{v}_{1}\right|} \\
& \times \sum_{n^{\prime} n} \mathcal{B}_{n^{\prime} n} P_{n_{f} l_{f} m_{f}}^{n^{\prime}}(\boldsymbol{s}),
\end{aligned}
$$

where $P_{n_{f} l_{f} m_{f}}^{n^{\prime} n}$ is the above-mentioned cubic polynomial which is extrapolated to the ionization threshold. Although only a finite number of $l_{f}$ values are calculated, transitions to final states with higher angular momenta (with $n_{f}>9$ and $l_{f}>6$ ) are insignificant because of the large centrifugal barrier experienced by those final states, which ensures that the overlap of the initial and final wave functions is small. Thus, the contributions of inelastic channels are included accurately in our calculations using the above-described procedures.

\section{B. Parameters of the incident electron pulse and the target}

The momentum amplitude $a_{0}\left(\boldsymbol{k}_{0}\right)$ of the incident electron pulse is modeled by a Gaussian distribution whose longitudinal and transverse widths are set, respectively, by the pulse duration and the angular spread of the pulse. The kinetic energy of the incident electron pulse in the laboratory frame is assumed to be $10 \mathrm{keV}$, which is one of energies for an 
attosecond electron pulse considered in Ref. [16]. For such a high energy the Born approximation is adequate to describe the collision process. The duration of our pulse is taken to be 100 as (FWHM), which is much shorter than the beat period, $\mathrm{T}=6.25 \mathrm{fs}$, of the target coherent state and provides sufficient temporal resolution. The angular spread of the pulse is chosen to be $\pm 10^{-4}$ rad. For the target, the kinetic energy is assumed to be its thermal energy at room temperature: $25 \mathrm{meV}$.

\section{Results for ultrafast electron pulse diffraction from coherent states of the $\mathbf{H}$ atom}

In Sec. III C 1 we present simulations for a target coherent state comprising an equal superposition of $\mathrm{H}$ atom $3 p$ and $4 p$ states. This equal-parity superposition involves a time-dependent "breathing" mode of electronic motion. In Sec. III C 2 we present simulations for a target coherent state comprising an equal superposition of $\mathrm{H}$ atom $3 d$ and $4 f$ states. This opposite-parity superposition involves a time-dependent "wiggling" mode of electronic motion. The time-dependent electron charge densities for each of these two coherent superposition states of the target $\mathrm{H}$ atom are illustrated in Fig. 2.

\section{Equal-parity superposition: $3 p+4 p$}

Since one way to produce an equal superposition of the $3 p$ and $4 p$ states of the $\mathrm{H}$ atom has been presented in detail in Ref. [19], we only summarize it briefly here. The key idea is to excite the $\mathrm{H}$ atom from its ground $1 s$ state using a linearly polarized laser pulse (polarized along the $z$ axis) whose bandwidth and central frequency are chosen so that the populations of the $3 p$ and $4 p$ states are equal, while the populations of other excited states are negligibly small. This can be accomplished by a pulse of duration $8.7 \mathrm{fs}$ and central energy $12.45 \mathrm{eV}$. The target electronic wave function may thus be written as

$$
\psi_{1}(t)=\frac{1}{\sqrt{2}}\left(\phi_{3 p} e^{-i \omega_{3 p} t}+\phi_{4 p} e^{-i \omega_{4 p} t}\right) .
$$

The electron charge density in the $y-z$ plane as a function of time is shown in the left-hand column of Fig. 2. The beat period $\mathrm{T}=2 \pi /\left(\omega_{4 p}-\omega_{3 p}\right)$ of the charge oscillation is $6.25 \mathrm{fs}$. One sees that the electron charge density exhibits a breathing mode of motion: It is compact and localized close to the nucleus at time zero and then expands with increasing time, reaching a maximum average radius at $t=\mathrm{T} / 2$, before receding back to the initial charge density distribution at time zero. The average radius of this charge density in the $\mathrm{H}$ atom oscillates from 14 to 22 a.u. Because this is an equal-parity superposition, the spatial symmetry of the charge distribution is unchanged as a function of time.

In Fig. 3 we present differential probabilities $d \mathscr{P} / d \hat{\boldsymbol{k}}_{a}$ [calculated using Eq. (37) and the results in Sec. III A] for scattering of a 100-as, $10-\mathrm{keV}$ electron pulse from a coherent, equal superposition of the $3 p$ and $4 p$ states of the $\mathrm{H}$ atom for three pump-probe delay times $t_{d}$, i.e., the time between the production of the coherent target state and the arrival of the electron pulse at the target. Note that the differential probabilities are evaluated in the center-of-mass frame, although only small differences are introduced in

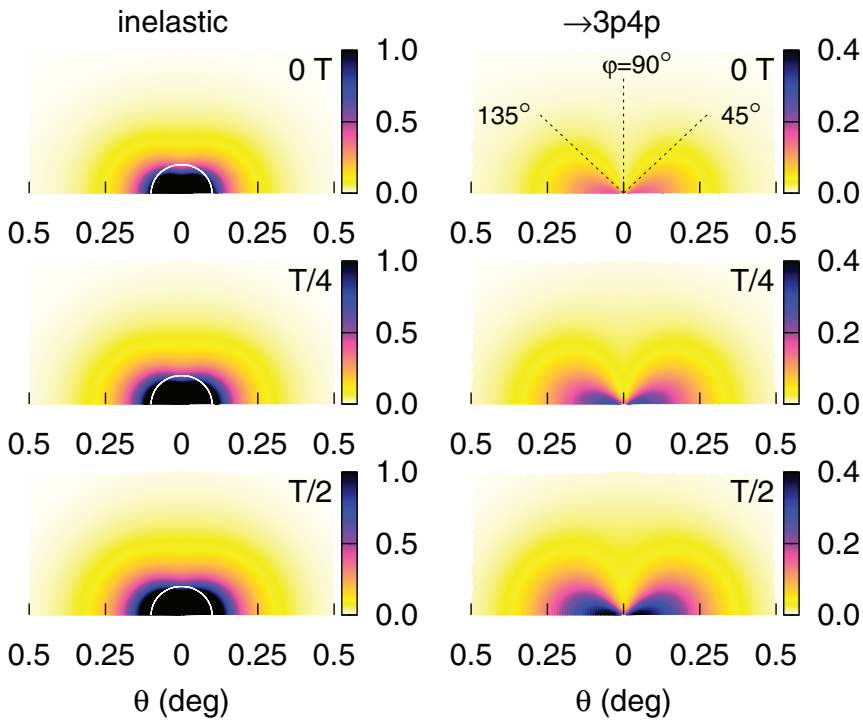

FIG. 3. (Color online) The inelastic (left column) and the restricted (right column) differential probabilities for scattering of a 100 -as, 10-keV electron pulse from a coherent, equal superposition of the $3 p$ and $4 p$ states of the $\mathrm{H}$ atom for three pump-probe delay times $t_{d}: 0 \mathrm{~T}, \mathrm{~T} / 4$, and $\mathrm{T} / 2$. Owing to symmetry, only the upper half of the images are shown. The white half circles along $\theta=0.1^{\circ}$ in the left column enclose forward scattering angles in which inelastic transitions dominate. See text for discussion and Fig. 1 for the definitions of the angles $\theta$ and $\varphi$. Note that the "inelastic" results in the left column include all significant inelastic transitions of the coherent $3 p+4 p$ state to $\mathrm{H}$ atom bound states, whereas the "restricted" results in the right column only contain inelastic transitions from the coherent $3 p+4 p$ state to the individual $3 p$ and $4 p$ states.

transforming to the laboratory frame owing to the heavy mass of the target relative to that of the incident electron. The left column of Fig. 3 presents the "inelastic" differential probabilities, i.e., the result of summing over bound final states $m$ of the target, as discussed in Sec. III A 2. For comparison, in the right column of Fig. 3 we present the "restricted" differential probabilities, which we define as the result of only summing over the $3 p$ and $4 p$ final states. These quasielastic restricted differential probabilities are pertinent to imaging the electronic motion of the initial coherent state of the target, because they exclude inelastic transitions to all other final target states besides $3 p$ and $4 p$.

As the delay time $t_{d}$ increases, the time dependence of both the inelastic and the restricted differential probabilities can be clearly observed, reflecting the target electronic motion. More specifically, the scattering intensities in both simulations increase as the charge density of the target wave function expands (cf. Fig. 2). The infinitely many inelastic channels included in the final-state sum in the case of the inelastic differential probabilities do not obscure the electronic motion, although their contributions to the scattering images add to the difficulty of retrieving details of the electronic motion and the target structure. However, the major differences between the inelastic and the restricted differential probabilities in Fig. 3 are actually localized in a narrow forward scattering cone, indicated by the white half circles in the panels of the left column. The predominance of the inelastic channels in the 
forward direction is anticipated, since, for hydrogenic atoms, the inelastic cross sections typically have a strong peak at small scattering angles, but decrease rapidly as the momentum transfer increases [30]. In contrast, for small scattering angles the restricted differential probabilities show a distinctive angular structure, due to the fact that only $3 p$ and $4 p$ states are in the final-state sum and both states have wave functions with the same angular dependence, $Y_{10}\left(\hat{\boldsymbol{y}}_{1}\right)$. One notices also a resemblance of the restricted differential probabilities in Fig. 3 with those in Fig. 3 of Ref. [19] for scattering from the charge distribution of the same target coherent state. However, the two results are obtained using two very different theoretical approaches: the coherent scattering description of this paper and the potential scattering description of Ref. [19]. Thus, despite the similarity of the results, one cannot assume that in the present calculations we are imaging the time-dependent target charge distribution.

In order to demonstrate the similar information provided by the inelastic and the restricted differential probabilities in Fig. 3, we present those results in Fig. 4 as functions of the azimuthal angle $\varphi$ at four fixed scattering angles, $\theta=0.3^{\circ}, 0.5^{\circ}, 1.0^{\circ}$, and $1.5^{\circ}$, and three different delay times. Note that these scattering angles $\theta$ avoid the largest contributions of the inelastic channels, which dominate the forward scattering differential probabilities for $\theta<0.3^{\circ}$. The angular dependencies of the differential probabilities in both simulations agree well, except for the greater magnitudes of the inelastic curves (due to the contributions from inelastic transitions to target final states other than $3 p$ and $4 p$ ). Three kinds of temporal behavior appear in both the inelastic and the restricted differential probabilities, which indicates that the inelastic channels do not alter the temporal behaviors
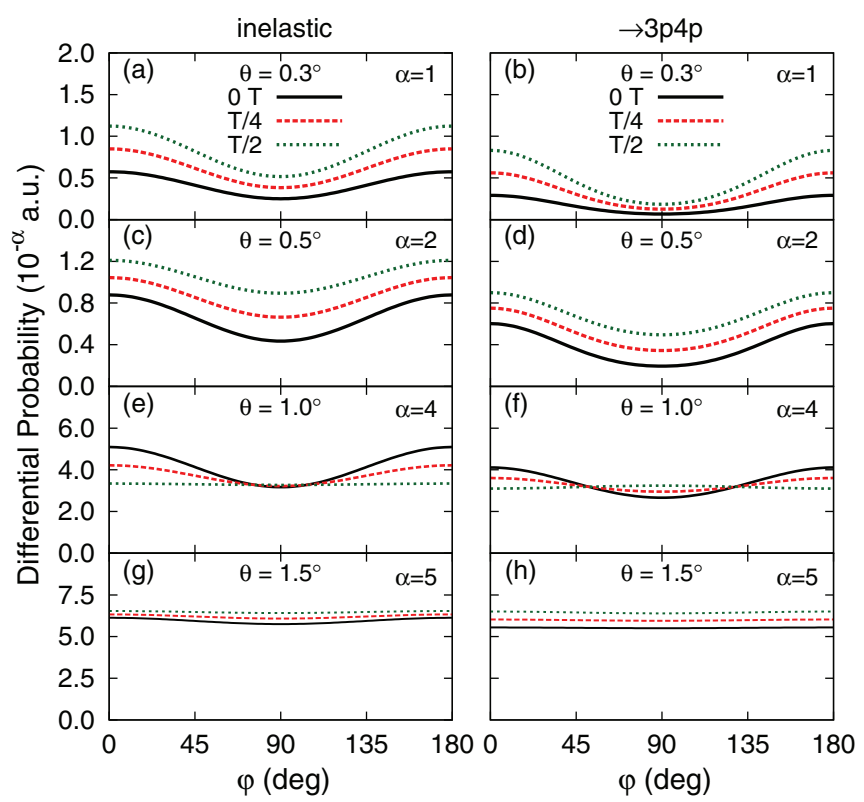

FIG. 4. (Color online) Comparison of the inelastic (left column) and the restricted (right column) differential probabilities as a function of azimuthal angle $\varphi$ for four fixed scattering angles, $\theta=0.3^{\circ}, 0.5^{\circ}$, $1.0^{\circ}$, and $1.5^{\circ}$, and three different pump-probe delay times, $t_{d}=0 \mathrm{~T}$, $\mathrm{T} / 4$, and $\mathrm{T} / 2$. at these scattering angles. In the first kind of temporal behavior, the overall scattering intensities at $\theta=0.5^{\circ}$ and $1.5^{\circ}$ increase by about the same magnitude at every $\varphi$ as $t_{d}$ increases, whereas in the second kind of temporal behavior the curve at $\theta=1.0^{\circ}$ oscillates as a function of $t_{d}$. Both kinds of behaviors can be explained as follows: In the restricted differential probabilities, the initial and final states have only $p$ orbital angular momentum; therefore, two terms $(l=0$ and 2) appear in the sum of Eq. (38), with the $l=0$ term having an isotropic distribution, while the $l=2$ term has a cosine-squared distribution. Moreover, since the transition matrices $T_{f i}$ (or the scattering amplitudes) from these two states are real [cf. Eqs. (39) and (40)], the interference terms in $d \mathscr{P} / d \hat{\boldsymbol{k}}_{a}$, which give the time dependence, behave like $\cos \left(\omega_{4 p}-\omega_{3 p}\right) t_{d}$. Hence, at $\theta=0.5^{\circ}$ and $1.5^{\circ}$ [Figs. 4(d) and 4(h)], the $l=0$ term dominates, so the curves increase rather uniformly over $\varphi$, whereas at $\theta=1.0^{\circ}$ [Fig. 4(f)], the $l=2$ term dominates, so the curve oscillates with the cosine-squared behavior. At $\theta=0.3^{\circ}$, these two terms give comparable contributions to the restricted differential probability, so that the third kind of temporal behavior [Fig. 4(b)] has a hybrid character.

To compare the inelastic and the restricted differential probabilities quantitatively, we present in Fig. 5 the ratios of these two differential probabilities for four fixed values of the scattering angle $\theta$. First, for every scattering angle, the ratio decreases as $t_{d}$ increases (in the range $0 \mathrm{~T} \leqslant t_{d} \leqslant \mathrm{~T} / 2$ ). Whereas the differential probabilities in Fig. 4 exhibited three kinds of temporal behavior at different scattering angles $\theta$, their

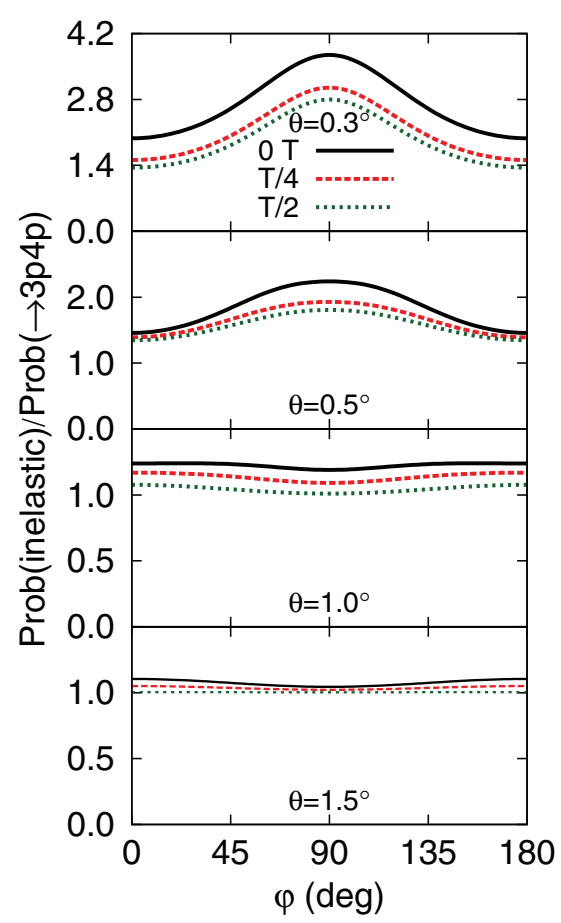

FIG. 5. (Color online) The ratios of the inelastic to the restricted differential probabilities as a function of $\varphi$ for four fixed scattering angles, $\theta=0.3^{\circ}, 0.5^{\circ}, 1.0^{\circ}$, and $1.5^{\circ}$, and three different pump-probe delay times, $t_{d}=0 \mathrm{~T}, \mathrm{~T} / 4$, and $\mathrm{T} / 2$. Note that the ordinate scale of the top two panels differs from that of the bottom two panels. 


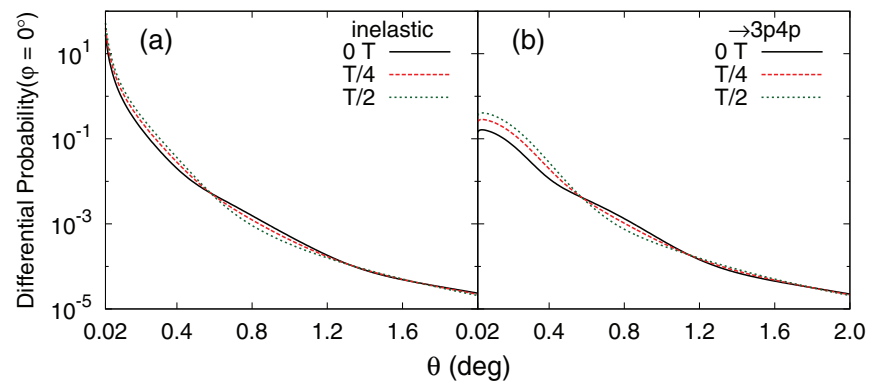

FIG. 6. (Color online) Comparison of (a) inelastic with (b) restricted differential probabilities as a function of the scattering angle $\theta$ for three different pump-probe delay times. In both panels, the azimuthal angle is $\varphi=0^{\circ}$.

ratios in Fig. 5 exhibit a single temporal behavior. Second, the ratios decrease and approach a constant value as $\theta$ increases, which follows from the fact that the inelastic channels are most significant at small scattering angles $\theta$ and their contribution at large scattering angles is rather isotropic along the azimuthal angle $\varphi$. The humps of the curves at $\theta=0.3^{\circ}$ and $0.5^{\circ}$ indicate that, although the differential probabilities in Figs. 4(a)4(d) exhibit concave shapes, the inelastic ones have smaller curvature. Moreover, the ratios of the differential probabilities around $\varphi=90^{\circ}$ are easily affected by the inelastic channels, owing to the small scattering probabilities of the restricted differential probabilities at $\varphi=90^{\circ}$ (cf. Fig. 4). However, at the other two scattering angles $\left(\theta=1.0^{\circ}\right.$ and $\left.1.5^{\circ}\right)$ the ratios show only a slight concave shape and take a value close to unity. In other words, at large scattering angles $\theta$ the differential probabilities reflect the electronic structure without significant complications stemming from inelastic channels other than $3 p$ and $4 p$.

In Fig. 6 we compare the inelastic and the restricted differential probabilities as a function of the scattering angle $\theta$ at $\varphi=0^{\circ}$. This figure essentially depicts the radial dependence of the differential probabilities [see Eq. (38) as well as Eq. (41) for the relation between $|\boldsymbol{s}|$ and $\theta$ ]. Observe first that the inelastic differential probabilities have a strong peak at small scattering angles, which is the signature of inelastic transitions, while the restricted ones are rather flat. (Note that as $\theta \rightarrow 0^{\circ}$, the transition amplitudes for $p \rightarrow p$ transitions must vanish according to electric dipole selection rules, but averaging over the angular divergence of the incident electron beam prevents the restricted differential probability from vanishing.) Observe also that the curves in the two panels become increasingly similar as the scattering angle increases. Second, we observe three nodes in each panel, and their positions correspond roughly to one another. This feature comes from the nodal behavior of the radial parts of the initial target wave function [30]. The three nodes in the differential probabilities result from the single radial node of the $3 p$ state and the two radial nodes of the $4 p$ state. Therefore, despite the strong forward peak in the inelastic differential probability, information on the nodal structures of the $3 p$ and $4 p$ states still can be obtained by studying the radial dependence of $d \mathscr{P} / d \hat{\boldsymbol{k}}_{a}$ [cf. Eqs. (38) and (41)].

The effects of the pulse duration of the projectile electron on the differential probabilities are shown in Fig. 7. Results

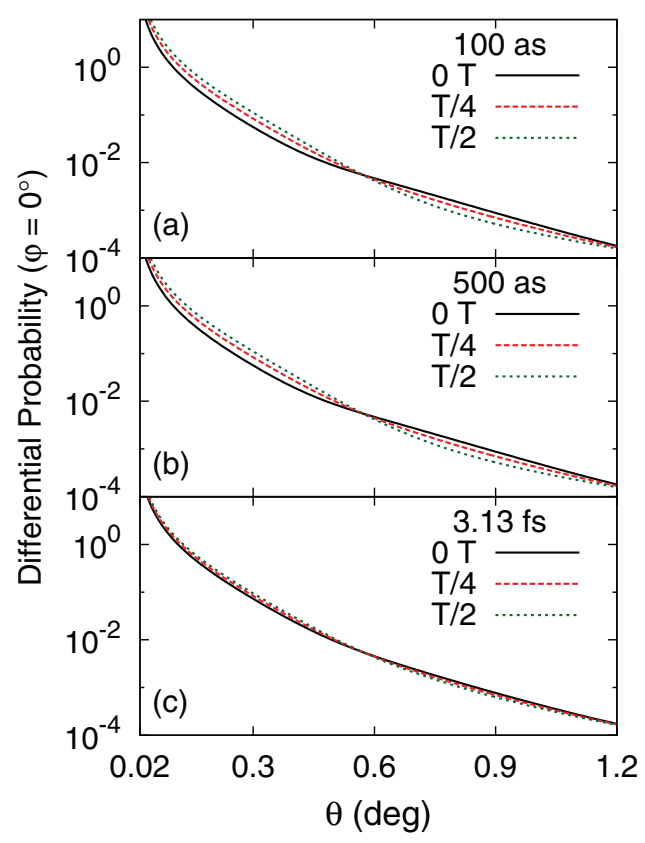

FIG. 7. (Color online) Inelastic differential probabilities for three different incident electron pulse durations (FWHM): (a) 100 as, (b) 500 as, and (c) 3.13 fs. Results for three different time delays are given in each panel for a fixed azimuthal angle, $\varphi=0^{\circ}$. Note that the differential probabilities presented in this figure do not include inelastic transitions to high Rydberg states with $n_{f}>9$; see text for discussion.

for three pulse lengths (FWHM) are shown-(a) 100 as, (b) 500 as, and (c) $3.13 \mathrm{fs-with} \mathrm{the} \mathrm{latter} \mathrm{equal} \mathrm{to} \mathrm{half} \mathrm{the}$ beat period $T$ of the initial target coherent state. Note that in these results only final states with $n_{f} \leqslant 9$ are included; i.e., no extrapolation of higher Rydberg levels has been carried out. (Comparing our 100-as results in Fig. 6, which include high Rydberg states, with those in Fig. 7, which do not, we find that the difference is less than $4 \%$ for all angles $\theta$. Please note the different ranges of the $\theta$ axis in these two figures.) The estimated energy bandwidths of the 100-as, 500-as, and 3.31-fs pulses are 18.2, 3.65, and $0.58 \mathrm{eV}$, respectively. Note first that there is no appreciable difference between the differential probabilities $d \mathscr{P} / d \hat{\boldsymbol{k}}_{a}$ for durations of 100 and 500 as. Since the approximations in Eqs. (22) and (27) are not used in our simulations, this insensitivity to pulse duration means that the factorization of the differential probability into target and electron pulse factors is a good approximation. Specifically, for incident electron pulses having appropriate temporal resolution, the character of the target structure can be factored out from the wave-packet integrals, and then the differential probabilities directly reflect the electronic motions of the targets [cf. Eq. (26)]. Note second that in contrast to Figs. 7(a) and 7(b), the temporal variation of the differential probability for the 3.13 -fs pulse is greatly diminished. In this case the electron pulse duration is too long to resolve the electronic motions and the energy bandwidth of the pulse is smaller than the energy difference of the target $3 p$ and $4 p$ states $(0.66 \mathrm{eV})$. As discussed in Sec. II above, if the pulse duration is much longer than the beat period of the charge oscillation, only the populations on each constituent state can 
be measured [cf. Eq. (30)]. For a coherent superposition state, the population on each of the eigenstates is constant. Therefore, the scattering patterns become independent of $t_{d}$ as the duration of the electron pulses becomes longer. Note finally that our results are inconsistent with the findings in Ref. [21] (for the case of an x-ray pulse scattered from a coherent target state), in which the contrast of the $\mathrm{x}$-ray pulse scattering signal as a function of time delay decreases as the pulse duration becomes shorter (see Fig. 5 A in [21]). Dixit et al. [21] conclude that the scattering pattern becomes independent of the target electron dynamics as the pulse duration approaches zero and that there is an optimal pulse duration for resolving target structure. The bandwidth of the $\mathrm{x}$-ray pulses in their simulations is about $0.5 \mathrm{eV}$.

Regardless of the temporal resolution, the contributions from the inelastic channels are at similar levels for all three pulse lengths. Thus, the radiation damage to the electronic structures appears to be only weakly dependent on the pulse duration. In contrast, for the case of x-ray scattering to image nanostructures, Chapman et al. [34] used short, intense $\mathrm{x}$-ray pulses to avoid the effects of radiation damage to the target on the x-ray scattering signal. They showed that the structure can be mapped before the sample is completely destroyed by an intense femtosecond $\mathrm{x}$-ray pulse and that the phase retrieval algorithm successfully reconstructs the molecular target structure. This is because the time scale for the thermalization of the energy deposited on the sample is 10$100 \mathrm{fs}$. If the pulse is much shorter than such a time scale, the $\mathrm{x}$-ray scattering is completed before the onset of the damage. In order to image target electronic structures, however, since the energy of an incident $\mathrm{x}$-ray or electron pulse is directly deposited on the target's electronic degrees of freedom, reducing the pulse length has no significant effect on the radiation damage for the small atomic system considered in this paper.

\section{Opposite-parity superposition: $3 d+4 f$}

In this second case we demonstrate a different type of target electronic motion, wiggling, which can be achieved by synthesizing a coherent superposition of opposite-parity $3 d$ and $4 f$ states of the $\mathrm{H}$ atom with angular momentum projection of zero on the $z$ axis. This state has been considered recently in Refs. [21,35]. The right column of Fig. 2 depicts the charge oscillation of this state from one side of the nucleus to the other. The charge density exhibits a complex angular dependence owing to the fact that the superposed states have high orbital angular momenta, $d$ and $f$. However, the charge density has a nodeless radial structure. Although the average radius of the coherent state is a constant, the individual $3 d$ and $4 f$ states have different average radii. Moreover, the coherent state is not stationary. Note that the $3 p+4 p$ and $3 d+4 f$ coherent states have the same beat period.

All parameters used in the present simulations are the same as those used previously for the $3 p+4 p$ case, except that the maximum orbital angular momentum is extended to $l_{f}=9$ in the extrapolation procedure (for summing over high Rydberg final states of the target) owing to the higher orbital angular momenta in the $3 d+4 f$ coherent state. In Fig. 8 we compare the inelastic and restricted differential probabilities
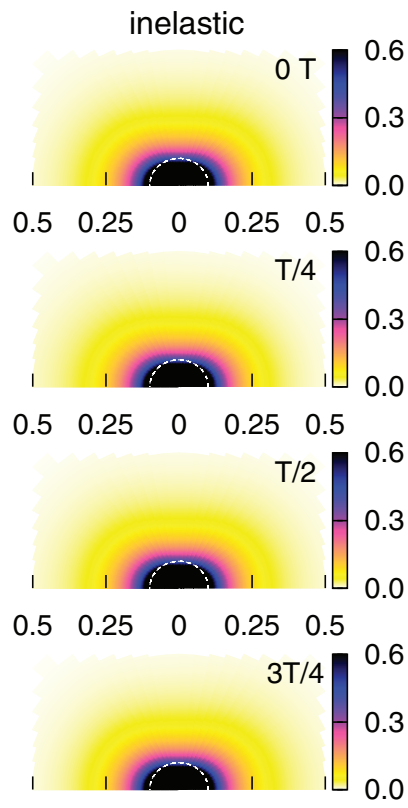

$\begin{array}{lllll}0.5 & 0.25 & 0 & 0.25 & 0.5\end{array}$ $\theta(\mathrm{deg})$
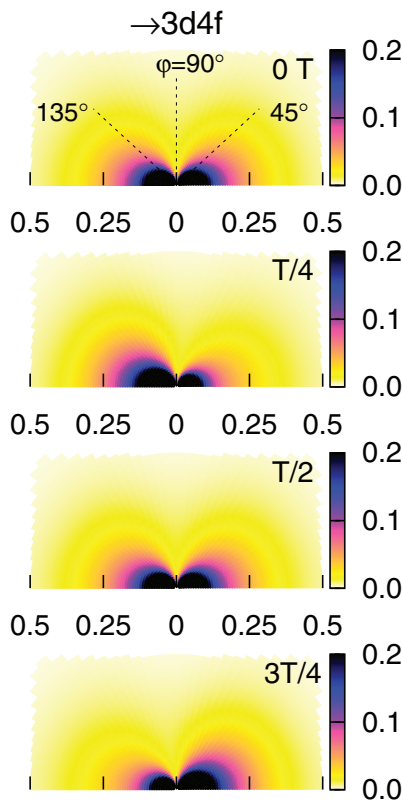

$\begin{array}{lllll}0.5 & 0.25 & 0 & 0.25 & 0.5\end{array}$

$\theta$ (deg)

FIG. 8. (Color online) The inelastic (left column) and the restricted (right column) differential probabilities for scattering of a 100 -as, 10-keV electron pulse from a coherent, equal superposition of the $3 d$ and $4 f$ states of the $\mathrm{H}$ atom for four pump-probe delay times $t_{d}: 0 \mathrm{~T}, \mathrm{~T} / 4, \mathrm{~T} / 2$, and $3 \mathrm{~T} / 4$. Owing to symmetry, only the upper half images are shown. The white half-circles along $\theta=0.1^{\circ}$ in the left column enclose forward scattering angles at which inelastic transitions dominate. The restricted differential probabilities include only $3 d$ and $4 f$ states in the sum over target final states $m$. See Fig. 1 for the definitions of the angles $\theta$ and $\varphi$.

for small forward scattering angles $\theta$ and four pump-probe delay times $t_{d}$. The most prominent feature of the restricted scattering images is the asymmetric scattering pattern with respect to $\varphi=90^{\circ}$ at $t_{d}=\mathrm{T} / 4$ and $3 \mathrm{~T} / 4$, which reflects the wiggling motion of the charge density. As for the $3 p+4 p$ case, inelastic channels greatly influence the images for small scattering angles $\theta \leqslant 0.1^{\circ}$. In general, the inelastic scattering patterns show more diffuse angular behaviors, and the temporal variation of the images can barely be seen for small forward scattering angles. Furthermore, no asymmetry of the images is observed. For the $3 d+4 f$ case, the temporal behavior is more easily affected by the summation over final target states than was the case for the $3 p+4 p$ target state. Although the asymmetry appears in the elasticlike restricted scattering images, the images oscillate out of phase with the motion of the charge density. The asymmetric charge densities at $t_{d}=0 \mathrm{~T}$ and $\mathrm{T} / 2$ in Fig. 2 correspond to symmetric scattering patterns at those time delays in Fig. 8, whereas when the charge densities are symmetric at $t_{d}=\mathrm{T} / 4$ and $3 \mathrm{~T} / 4$, the scattering patterns at those times are asymmetric. The same correspondence between the time-dependent charge density for the $3 d+4 f$ target state and the scattering patterns was also observed in Ref. [21] for the case of $\mathrm{x}$-ray pulse scattering. This idiosyncrasy stems from the complex-valued nature of wave 
functions and the interference of the scattering amplitudes, as can be shown from Eq. (24).

Consider the factors $c_{n^{\prime}}^{*} c_{n} \exp \left[i\left(\omega_{n^{\prime}}-\omega_{n}\right) t_{d}\right] T_{m n^{\prime}}^{*} T_{m n}$ in Eq. (24) for the differential probability, in which we have given the time dependence of the coefficients $C_{n^{\prime}}^{*} C_{n}$ explicitly. In the restricted calculation, $m, n^{\prime}$, and $n$ are restricted to the $3 d$ and $4 f$ states. Also, the only terms dependent on time are those for $n^{\prime} \neq n$. The transition matrix elements $T_{m n}$ are real if $m=n$ and purely imaginary if $m \neq n$. This follows from the fact that they are products of radial and angular factors [cf. Eq. (38)], where the radial factor is purely real [cf. Eq. (40)], while the angular factor $\Theta_{l m}$ is purely real or imaginary depending on its rank $l$, owing to the leading factor $i^{l}$ in Eq. (39). The rank $l$ is an even integer if $m=n\left(\right.$ or $\left.l_{f}=l_{i}\right)$ and is an odd integer if $m \neq n$ (or $l_{f} \neq l_{i}$ ). (Recall that the magnetic quantum numbers of the $3 d$ and $4 f$ states are zero.) Therefore, in the time-dependent terms $\left(n^{\prime} \neq n\right)$ in Eq. (24) the product of transition amplitudes $T_{m n^{\prime}}^{*} T_{m n}$ is purely imaginary since one of the amplitudes is purely real and the other is purely imaginary. Therefore, in the sum over $n^{\prime}$ and $n$ in Eq. (24) the terms $T_{m n^{\prime}}^{*} T_{m n}$ and $T_{m n}^{*} T_{m n^{\prime}}$ have opposite signs, so that the real part of the time-dependent factor $\exp \left[i\left(\omega_{n^{\prime}}-\omega_{n}\right) t_{d}\right]$ does not contribute to the sum; only its imaginary part, $\propto \sin \left(\omega_{n^{\prime}}-\omega_{n}\right) t_{d}$ contributes and this time-dependent part vanishes for $t_{d}=0 \mathrm{~T}$ and $\mathrm{T} / 2$. This term (a)

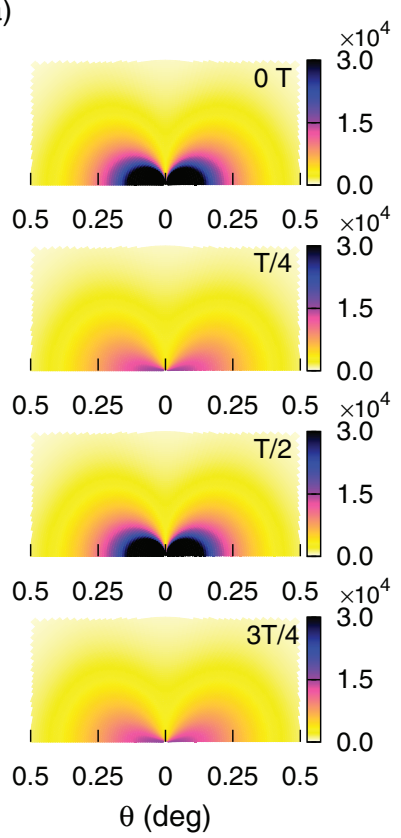

(b)

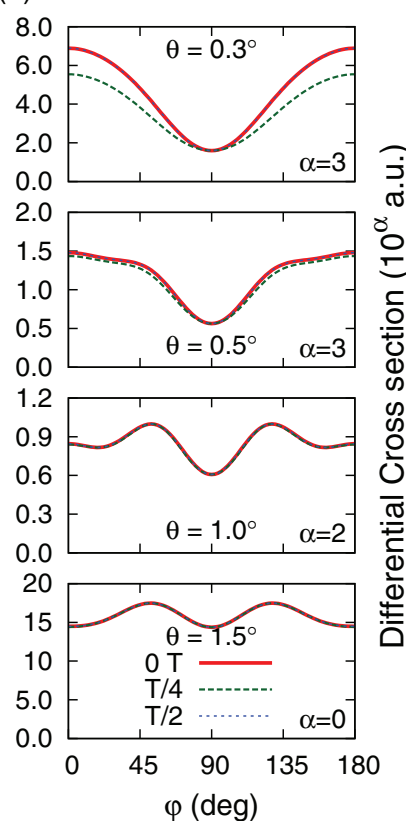

FIG. 9. (Color online) Differential cross sections for scattering of a 100-as, 10-keV electron pulse from the charge density of an equal superposition of the $3 d$ and $4 f$ states of the $\mathrm{H}$ atom, calculated according to Eq. (1) without averaging over the momentum profile of the incident electron pulse. Column (a): Differential cross sections for four pump-probe delay times $t_{d}: 0 \mathrm{~T}, \mathrm{~T} / 4, \mathrm{~T} / 2$, and 3T/4. Column (b): Differential cross sections as a function of the azimuthal angle $\varphi$ for four fixed scattering angles: $\theta=0.3^{\circ}, 0.5^{\circ}, 1.0^{\circ}$, and $1.5^{\circ}$. Note that results for $t_{d}=0 \mathrm{~T}$ and $\mathrm{T} / 2$ are equal, as are those for $\mathrm{T} / 4$ and $3 \mathrm{~T} / 4$, and all results are symmetric about $\theta=0$. These temporal and spatial symmetries are very different from those calculated using Eq. (37), which are shown in Fig. 8. does not vanish when $t_{d}=\mathrm{T} / 4$ and $3 \mathrm{~T} / 4$ and the spherical harmonic $Y_{l 0}^{*}(\hat{\boldsymbol{s}})$ in the angular factor [cf. Eq. (39)] for the imaginary transition amplitude (having odd order $l$ ) gives the asymmetric angular distribution shown in Fig. 8.

If the charge density, and not the wave function, is used to calculate the differential cross sections [using Eq. (1)], one obtains the results shown in Fig. 9. The differential cross sections are centrosymmetric at all delay times, which is very different from the temporal and spatial symmetry of the charge density itself, shown in the right column of Fig. 2, and also very different from the results of a proper calculation of the differential probabilities according to Eq. (37), which are shown in Fig. 8. In particular, although the results of a proper calculation are not easily related to the time-dependent charge density, they do show the symmetries and time-dependent wiggling motion of the charge density.

Since it is difficult to discern the time dependence of the differential probability $d \mathscr{P} / d \hat{\boldsymbol{k}}_{a}$ in the case when inelastic final-state transitions are included (cf. the left column of Fig. 8), in Fig. 10 we compare the inelastic and the restricted differential probabilities along the $z$ axis $\left(\varphi=0^{\circ}\right.$ and $\left.180^{\circ}\right)$ at different delay times as functions of the scattering angle $\theta$ in order to examine the effects of the inelastic channels at large momentum transfers [cf. Eq. (41)]. The results for $t_{d}=\mathrm{T} / 2$ are not shown, because they are identical to those at zero delay time. Comparing the inelastic with the restricted differential probabilities, one sees that the inelastic channels affect the differential probabilities over a wide range of scattering angles $\theta$. This is different from the previous $3 p+4 p$ case in which at large momentum transfer the contributions of the inelastic channels diminish. Nevertheless, the inelastic differential
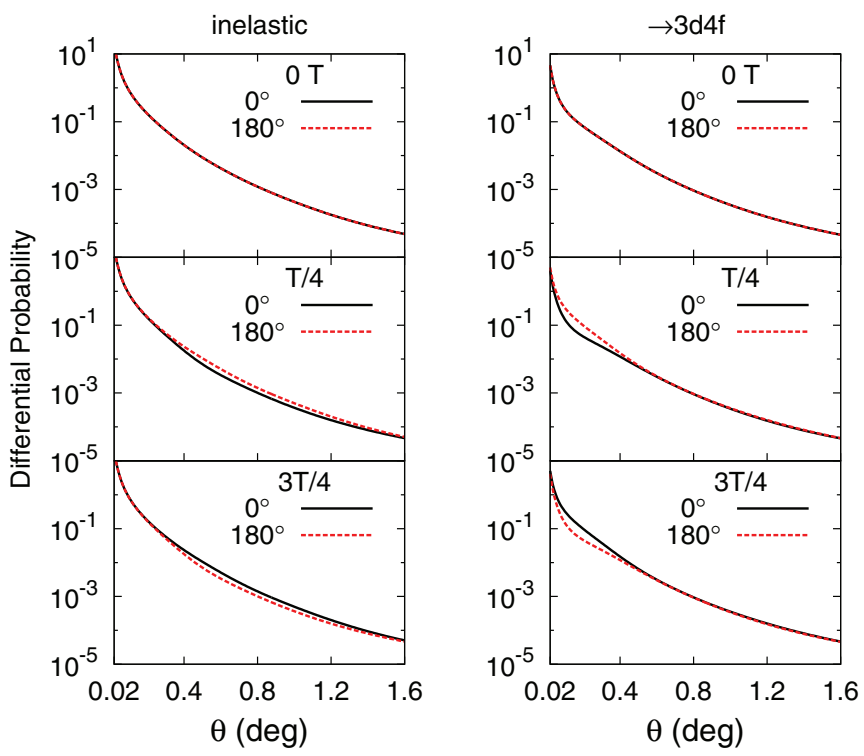

FIG. 10. (Color online) The inelastic (left column) and the restricted (right column) differential probabilities for scattering of a 100 -as, 10-keV electron pulse from a coherent, equal superposition of the $3 d$ and $4 f$ states of the $\mathrm{H}$ atom as a function of scattering angle $\theta$ for two fixed azimuthal angles: $\varphi=0^{\circ}$ (positive $z$ axis) and $\varphi=180^{\circ}$ (negative $z$ axis). Results are shown for three pump-probe delay times: $t_{d}=0 \mathrm{~T}, \mathrm{~T} / 4$, and $3 \mathrm{~T} / 4$. Results for $t_{d}=\mathrm{T} / 2$ are identical to those for $t_{d}=0 \mathrm{~T}$. 
probabilities in Fig. 10 do show a clear time dependence over a broad range of angles $\theta$. Indeed, the difference between the inelastic curves at $\varphi=0^{\circ}$ and $180^{\circ}$ for $t_{d}=\mathrm{T} / 4$ or $3 \mathrm{~T} / 4$ manifests the asymmetric scattering patterns corresponding to the wiggling motion of the target coherent state, even at large momentum transfers. In the case of the restricted differential probabilities, however, the temporal variations are confined to scattering angles $\theta<0.6^{\circ}$. Unlike the case of the $3 p+4 p$ target state (cf. Fig. 6), neither of the differential probabilities in Fig. 10 shows oscillations in the $\theta$ dependence of the asymmetries along the positive and negative $z$ axis, reflecting the nodeless radial wave functions of the constituents of the $3 d+4 f$ target coherent state. The restricted scattering images show a narrow spike close to zero scattering angle, which did not appear in the restricted differential probability for the $3 p+4 p$ case [cf. Fig. 6(b)]. This is because the transition between the $3 d$ and $4 f$ states is a dipole-allowed transition, so that even excluding other inelastic channels, which usually peak in the forward direction, the dipole-allowed transition leads to a spike as $\theta \rightarrow 0^{\circ}$.

In Fig. 11 we compare the inelastic and restricted differential probabilities as functions of the azimuthal angle $\varphi$ at four fixed scattering angles: $\theta=0.3^{\circ}, 0.5^{\circ}, 1.0^{\circ}$, and $1.5^{\circ}$. Observe first that the overall shapes of the inelastic curves at $t_{d}=0 \mathrm{~T}$ agree with the restricted ones at all scattering angles $\theta$. As for the case of the $3 p+4 p$ target state, the inelastic curves in Fig. 11 are shifted upward relative to the restricted curves due to inelastic contributions. Therefore, except for the asymmetric oscillations seen for time delays $t_{d}=\mathrm{T} / 4$ and $3 \mathrm{~T} / 4$, the inelastic channels contribute rather isotropically to

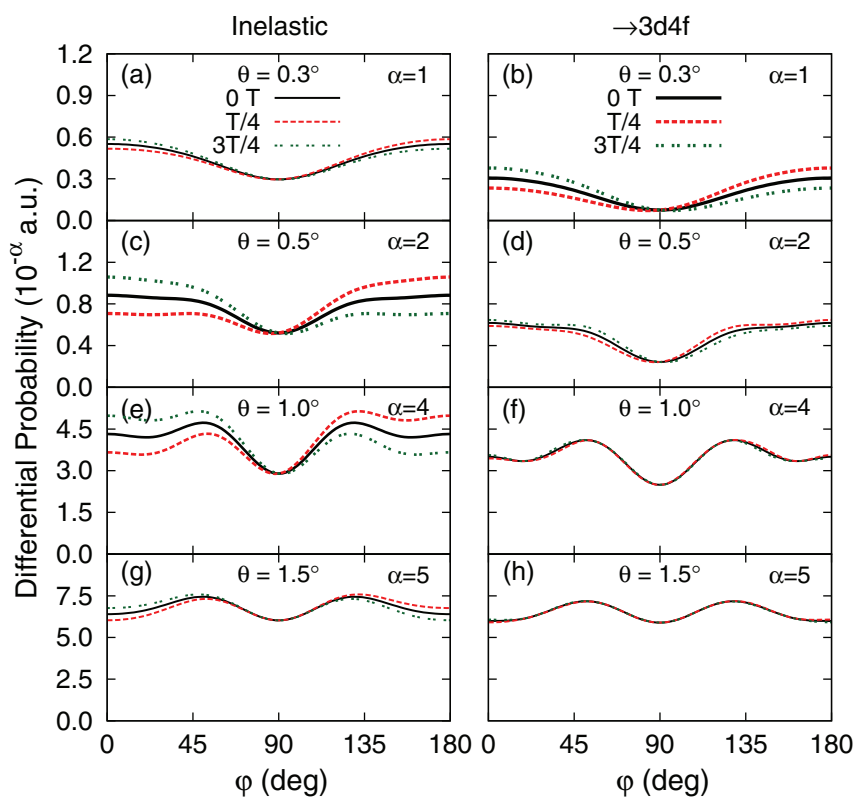

FIG. 11. (Color online) The inelastic (left column) and the restricted (right column) differential probabilities for scattering of a 100 -as, $10-\mathrm{keV}$ electron pulse from a coherent, equal superposition of the $3 d$ and $4 f$ states of the $\mathrm{H}$ atom as a function of the azimuthal angle $\varphi$ at four fixed scattering angles: $\theta=0.3^{\circ}, 0.5^{\circ}, 1.0^{\circ}$, and $1.5^{\circ}$. Results are shown for three pump-probe delay times: $t_{d}=0 \mathrm{~T}, \mathrm{~T} / 4$, and $3 \mathrm{~T} / 4$. Results for $t_{d}=\mathrm{T} / 2$ are identical to those for $t_{d}=0 \mathrm{~T}$. the differential probabilities at large scattering angles. Second, observe that the oscillating angular dependence seen in the inelastic curves for $\theta=1.0^{\circ}$ and $1.5^{\circ}$ indicates that high orbital angular momenta are present in the initial target coherent superposition state. Finally, the see-saw oscillation of the curves as a function of $t_{d}$ implies that no apparent changes in the total differential probabilities are observed at different delay times. This is expected because the charge density just wiggles from one side to the other as a function of time and the average radius of the coherent state is constant during this oscillation. This wiggling charge oscillation is reflected in the asymmetry of the scattering images, rather than in the overall scattering intensities. Moreover, inclusion of the inelastic transitions appears to amplify the magnitude of these oscillations, thus making them more easily detectable.

\section{SUMMARY AND CONCLUSIONS}

In summary, we have developed a scattering theory to describe the collision of ultrafast electron pulses from coherent superposition states of atomic targets. The theoretical analysis shows that the longitudinal coherence of the electron pulses affects the kinds of information concerning time-dependent target electronic motions that can be obtained from diffraction experiments. In our simulations, we have investigated the patterns in the differential scattering probabilities produced by attosecond electron pulses scattered from the breathing and wiggling modes of electronic motion arising from the beating of the $3 p+4 p$ and $3 d+4 f$ coherent superposition states, respectively, of an $\mathrm{H}$ atom target. The simulation results for the differential scattering probabilities in both cases manifest clear time-dependent images, confirming the ability of attosecond electron pulses to image the electronic motions [3,18-20]. Moreover, the symmetry of the images and their temporal behaviors distinguish the two kinds of motion we have considered in this paper. The results also demonstrate that, under appropriate conditions, the factorization of the properties of the projectile electron and the target is achievable, and, in those cases therefore, the scattering images directly reflect the time-dependent target structures. However, for small targets (as in the present case of the $\mathrm{H}$ atom), the contributions of inelastic channels, and the consequent damage to the coherent electronic structures of the target, seems inevitable, and reducing the pulse duration has little effect.

Our theoretical analysis and numerical simulations do not support the interpretation of the scattering of an ultrafast electron pulse from a time-dependent coherent target state as the scattering from the time-dependent charge-density of the target, as in Eq. (1) (provided, of course, that the experiments are carried out as described in the theory presented in Sec. II). Accordingly, any algorithms aiming to retrieve target structures from the differential scattering probabilities must be appropriately modified in order to properly reconstruct the target electronic motions and structures. Nevertheless, the possibility of imaging the time-dependent charge densities is not excluded. For example, x-ray phase contrast imaging has been proposed to obtain the time-dependent charge densities without the complications introduced by inelastic scattering processes [35]. Also, time-resolved $(e, 2 e)$ momentum spectroscopy has been proposed as a means to image the 
momentum space charge densities of the initial states of electrons ionized from atoms and molecules [20].

Since only the $\mathrm{H}$ atom is involved in the present investigation, the generalization of our conclusions to multielectron targets remains an issue for further research. Moreover, we note that there exist factors that we have neglected which may affect the results of our simulations. For example, the unavoidable decoherence of the incident electron pulse as it travels toward the target has not been simulated. Moreover, the time-dependent electronic motions in more complex target systems cannot, in general, be simply characterized by the two kinds of motion considered in this paper. Nevertheless, our simulations do shed light on the potentiality and the issues involved in studying electron dynamics by means of ultrafast electron diffraction.

Although this work is specialized to atomic systems, the generalization to molecular targets is straightforward by including the ro-vibrational degrees of freedom. Novel diffraction patterns from molecular targets are expected, because of the interference of scattering amplitudes from more complicated electronic motions. With the anticipated future advances of ultrafast electron techniques, four-dimensional electron movies seem feasible for directly investigating the roles of electrons in atomic and molecular reactions by means of tabletop-scale experiments.

Finally, we note that detection of the scattered electron energy may provide more information on time-dependent target electronic motion. However, success in this regard depends on the energy level spectrum of the target, the duration of the electron pulse, and the time-scale of the target electronic motion. We plan to report on these aspects elsewhere.

\section{ACKNOWLEDGMENTS}

This work is supported in part by AFOSR Award No. FA9550-12-1-0149. Our calculations were carried out at the Holland Computing Center of the University of Nebraska.
[1] A. H. Zewail, J. Phys. Chem. A 104, 5660 (2000).

[2] J. R. Dwyer, C. T. Hebeisen, R. Ernstorfer, M. Harb, V. B. Deyirmenjian, R. E. Jordan, and R. J. D. Miller, Philos. Trans. R. Soc. A 364, 741 (2006).

[3] P. Baum and A. H. Zewail, Chem. Phys. 366, 2 (2009).

[4] P. Wernet, Phys. Chem. Chem. Phys. 13, 16941 (2011).

[5] P. Salières, A. Maquet, S. Haessler, J. Caillat, and R. Taïeb, Rep. Prog. Phys. 75, 062401 (2012).

[6] M. J. J. Vrakking and T. Elsaesser, Nat. Photon. 6, 645 (2012).

[7] P. Emma et al., Nat. Phot. 4, 641 (2010).

[8] G. Sciaini and R. J. D. Miller, Rep. Prog. Phys. 74, 096101 (2011).

[9] A. H. Zewail, Annu. Rev. Phys. Chem. 57, 65 (2006).

[10] D. Shorokhov and A. H. Zewail, Phys. Chem. Chem. Phys. 10, 2879 (2008).

[11] A. H. Zewail, Science 328, 187 (2010).

[12] D. J. Flannigan and A. H. Zewail, Acc. Chem. Res. 45, 1828 (2012).

[13] T. van Oudheusden, P. L. E. M. Pasmans, S. B. van der Geer, M. J. de Loos, M. J. van der Wiel, and O. J. Luiten, Phys. Rev. Lett. 105, 264801 (2010).

[14] E. Fill, L. Veisz, A. Apolonski, and F. Krausz, New J. Phys. 8, 272 (2006).

[15] A. Gliserin, A. Apolonski, F. Krausz, and P. Baum, New J. Phys. 14, 073055 (2012).

[16] P. Baum and A. H. Zewail, Proc. Natl. Acad. Sci. USA 104, 18409 (2007).

[17] S. A. Hilbert, C. Uiterwaal, B. Barwick, H. Batelaan, and A. H. Zewail, Proc. Natl. Acad. Sci. USA 106, 10558 (2009).

[18] P. Baum, J. Manz, and A. Schild, Sci. China: Phys., Mech. Astron. 53, 987 (2010).
[19] H.-C. Shao and A. F. Starace, Phys. Rev. Lett. 105, 263201 (2010).

[20] H.-C. Shao and A. F. Starace, Phys. Rev. A 87, 050701(R) (2013).

[21] G. Dixit, O. Vendrell, and R. Santra, Proc. Natl. Acad. Sci. USA 109, 11636 (2012).

[22] J. C. H. Spence, in Science of Microscopy, edited by P. W. Hawkes and J. C. H. Spence (Springer, New York, 2007), Vol. II, p. 1196.

[23] F. Robicheaux, Phys. Rev. A 62, 062706 (2000).

[24] M. Inokuti, Rev. Mod. Phys. 43, 297 (1971).

[25] M. L. Goldberger and K. M. Watson, Collision Theory (Wiley \& Sons, New York, 1964).

[26] E. Merzbacher, Quantum Mechanics, 3rd ed. (Wiley \& Sons, New York, 1998).

[27] P. Baum, Chem. Phys. 423, 55 (2013).

[28] P. Baum and A. H. Zewail, Proc. Natl. Acad. Sci. USA 103, 16105 (2006).

[29] H. A. Bethe and R. Jackiw, Intermediate Quantum Mechanics (W. A. Benjamin, New York, 1968).

[30] R. A. Bonham and M. Fink, High Energy Electron Scattering (Van Nostrand Reinhold, New York, 1974).

[31] H. S. W. Massey and C. B. O. Mohr, Proc. R. Soc. A 132, 605 (1931).

[32] R. McCarroll, Proc. Phys. Soc. A 70, 460 (1957).

[33] G. W. F. Drake and R. N. Hill, J. Phys. B 26, 3159 (1993).

[34] H. N. Chapman et al., Nat. Phys. 2, 839 (2006).

[35] G. Dixit, J. M. Slowik, and R. Santra, Phys. Rev. Lett. 110, 137403 (2013). 